Research Article

\title{
Design of a Magnetic Interaction-Based Vibration Absorber for Continuous Beam
}

\author{
Guyue Jiao \\ School of Mechanical Engineering, University of Shanghai for Science and Technology, Shanghai, China \\ Correspondence should be addressed to Guyue Jiao; jiaoguyue@usst.edu.cn
}

Received 24 September 2019; Revised 21 March 2020; Accepted 14 May 2020; Published 22 June 2020

Academic Editor: Stefano Marchesiello

Copyright $\odot 2020$ Guyue Jiao. This is an open access article distributed under the Creative Commons Attribution License, which permits unrestricted use, distribution, and reproduction in any medium, provided the original work is properly cited.

\begin{abstract}
Classical absorber for vibration suppression of a continuous structure is constructed as a spring-mass oscillator, which only provides coupling force to suppress the vibration of primary structure. In this study, absorber beam is introduced and coupled on the continuous primary beam with magnetic interaction. Thus, the magnetic interaction and coupling bending moment affect the responses of primary beam. Based on the model of the system and Galerkin truncation, the natural frequencies for different magnetic parameters are obtained, which demonstrates that the fundamental frequency can be reduced to zero and the vibration of primary beam can be suppressed in a wide frequency band. Considering the vibration suppression on frequency band, we propose two criteria to evaluate the vibration suppression effect: one is the width of band for vibration suppression and the other is the width for vibration absorption. The two criteria not only show the vibration reduction effect but also correspond to different vibration suppression mechanism. Due to the advantages of zero fundamental frequency induced by the proposed magnetic interaction coupling and wide vibration suppression frequency band, utilizing absorber beam in vibration suppression of continuous structure has potential applications for flexible aim in the fields of manufacturing and aerospace.
\end{abstract}

\section{Introduction}

Due to urgent requirements and harsh conditions in the vibration suppression techniques in the fields of aerospace [1], shipping engineering [2], and ocean platforms [3, 4], it needs the remarkable vibration suppression effectiveness in a wide frequency band. For the vibration suppression and reduction of a vibration system, tuned vibration absorber (TVA) is designed according to different requirements in practices. Usually, the TVA is a single degree-of-freedom (1DOF) spring-damper-mass vibration oscillator designed with adjustable stiffness or damping $[5,6]$. When the targeted frequency for vibration suppression of the primary system is in the region of antifrequency band induced by the vibration absorber, the vibration energy can be transmitted effectively. However, in the analysis and applications of TVA, there are mainly two limitations and issues in design and realization. First, the vibration suppression effectiveness is strongly dependent on the structural parameters of the TVA, especially the mass. For smaller mass of TVA, the vibration suppression is less sufficient or effective. But, in the practices, the mass of absorber is required lighter than $10 \%$ mass of the primary system. Second, the stiffness and damping of the vibration absorber should have tunable property to result in antiresonance at the required frequencies for the realization of optimal vibration suppression effectiveness. When the primary system has ultralow natural frequency or variable characteristics, the structural realization of TVA is required to match the characteristics of primary system.

Recent studies of vibration absorption mainly focused on the bottleneck techniques for the realization of adjustable and adaptive properties. The methods include passive vibration device, active control methodology, and hybrid control devices. Based on the remarkable tunable properties for equivalent stiffness and damping of the so-called quasizero stiffness system [7-9], the structure is transplanted to the absorber structure for the required vibration absorption frequencies [10]. It also discovers that the vibration performances of nonlinear tuned vibration absorber (NTVA) 
may result in some beneficial dynamical phenomena in vibration suppression, such as saturation properties and internal resonances, which cannot exist for linear vibration systems [11-13]. Furthermore, for vibration suppression in a frequency band rather than just on specific frequency point, periodic structures are applied on continuous primary system and designed for wide bandgap based on local resonance theory $[14,15]$. Recently, in order to extend the bandgap to low frequency band, the QZS system is utilized as the TVA $[16,17]$. With the periodical assembly of oscillators with QZS property on a continuous beam, a low-frequency bandgap is realized due to the negative-stiffness structural design. But, with the reduction of bandgap to low frequency band, the width of bandgap reduces. On one hand, these studies are concentrated on the frequency tuning technologies by structural design to adapt to the targeted frequencies or frequency bands. On the other hand, the vibration amplitudes, especially at resonances, should be attended to and suppressed. Commonly, the most direct method to reduce the resonance peak is increasing the equivalent damping effect of the system. However, although increasing the equivalent damping strength can reduce the resonance peaks, the amplitudes at the nonresonance bands would be raised, inducing deterioration of vibration suppression. In order to harmonize the contradiction on the response amplitude, the most traditional method to design the TVA is the $H_{\infty}$ criterion to minimize the vibration amplitudes of the primary system around resonance band [18-21]. The optimal parameters obtained by the $H_{\infty}$ strategy work very well since the resonance peaks of the 2DOF system are minimum and equal [19-22]. Then, for continuous structure such as beam, the TVA is also designed as a beam, connected on the continuous primary beam by spring and damper [23]. Simplifying the model as a 2-DOF system and applying the fixed-point theory, the vibration of primary beam is reduced, but the vibration suppression is dependent on the mass ratio between the primary beam and absorber beam. In [24-26], an absorber beam with/without lumped mass is fixed on a primary beam on perpendicular position. The proposed structural assembly of the proposed absorber beam can achieve more vibration reduction with same mass ratio compared with the traditional springdamping-mass absorber.

From the previous studies, it can be seen that the structural design or control strategy for vibration suppression by both frequency tuning and amplitude reduction is well needed. In addition, it also requires lightweight TVA with appropriate nonlinearity. Thus, this study proposes an absorber beam with negative stiffness components, which is coupled on a primary beam. In the proposed structure, the tunable negative stiffness components are engendered by magnetic interaction. The negative stiffness components are two pairs of magnets with very small mass assembled symmetrically on the absorber beam and the free end of the primary beam, adjustable for different structural parameters. Due to the negative stiffness coupling, the stiffness and coupled stiffness of the absorber beam change cooperating with different equivalent stiffness of the primary beam. For vibration, the motion of the free end of the primary beam amplifies the coupling force in each modal, and thus the mass of the absorber beam can be very tiny. Since it has been verified that the nonlinear interaction force can suppress the vibration energy and reduce the resonance peaks [27-30], the nonlinearity introduced into the system can benefit the reduction of resonance frequency and amplitude reduction. Therefore, combining the advantages of linearity and nonlinearity, the absorber beam, with very small mass, can induce a wide nonresonance frequency band and low resonance peaks.

The paper is organized as follows. First, the model of magnetic interaction is established and its effect is discussed in Section 2. Next, the dynamical model of the nonlinear coupling system is established and all coefficients in the model are defined in Section 3. Then, based on the dynamical model, the natural frequencies and solutions are solved. Also, the evaluation criteria for effective vibration suppression are proposed in Section 4. The evaluation criteria provide conditions for mechanisms of vibration absorption and design method for structural parameters. The experimental setup and results are discussed in Section 5. The conclusion is given in the final Section.

\section{Structure of System Coupled by Magnetic Interaction}

2.1. Structure. Traditionally, for the vibration suppression of a continuous structure such as cantilever beam, springdamper-mass oscillator is applied on the cantilever beam as the TVA. Considering the moment can directly induce bending deformation of continuous beam, the absorber is designed to result in both force and moment interactions on the primary system in this study. Therefore, an absorber beam, which has much smaller mass than the primary beam, is introduced and coupled on the primary beam by constraint and magnetic interaction, as shown in Figure 1. All the structural parameters in the system are listed in Table 1 in Appendix.

As shown in Figure 1(a), the primary beam is excited by an excitation force $f(t)$ and the absorber beam is coupled with the primary beam under magnetic interaction. As shown in Figure 1(b), the vibrations of the primary beam and absorber beam are $w_{1}\left(\xi_{1}, t\right)$ and $w_{2}\left(\xi_{1}, t\right)$ with coordinates $\xi_{1}$ and $\xi_{2}$, respectively. Since the motion of free end of the primary beam is $w_{1}\left(L_{1}, t\right)$ and the angle of rotation is $\theta_{1}\left(L_{1}, t\right)$, the vibration motion of absorber beam consisted of three parts as shown in Figure 1(b), written as

$$
w_{2}\left(\xi_{2}, t\right)=w_{1}\left(L_{1}, t\right)+\theta_{1}\left(L_{1}, t\right) \xi_{2}+\widehat{w}_{2}\left(\xi_{2}, t\right) .
$$

In equation $(1), \widehat{w}_{2}\left(\xi_{2}, t\right)$ is the deflection vibration and $w_{1}\left(L_{1}, t\right)+\theta_{1}\left(L_{1}, t\right) \xi_{2}$ is the rigid displacement. The values of structural parameters are fixed as given in Table 1 in Appendix. As shown in Table 1, the mass of the primary beam is $\rho_{2} A_{2} L_{2}=0.0216 \mathrm{~kg}$, which is about 120 times the mass of absorber beam equal to $\rho_{2} A_{2} L_{2}=0.00018 \mathrm{~kg}$. By coupling the absorber beam with cross section constraint and magnetic interaction, the effect and mechanism of absorber beam are estimated in this study. The model of magnetic interaction is established in the following section. 


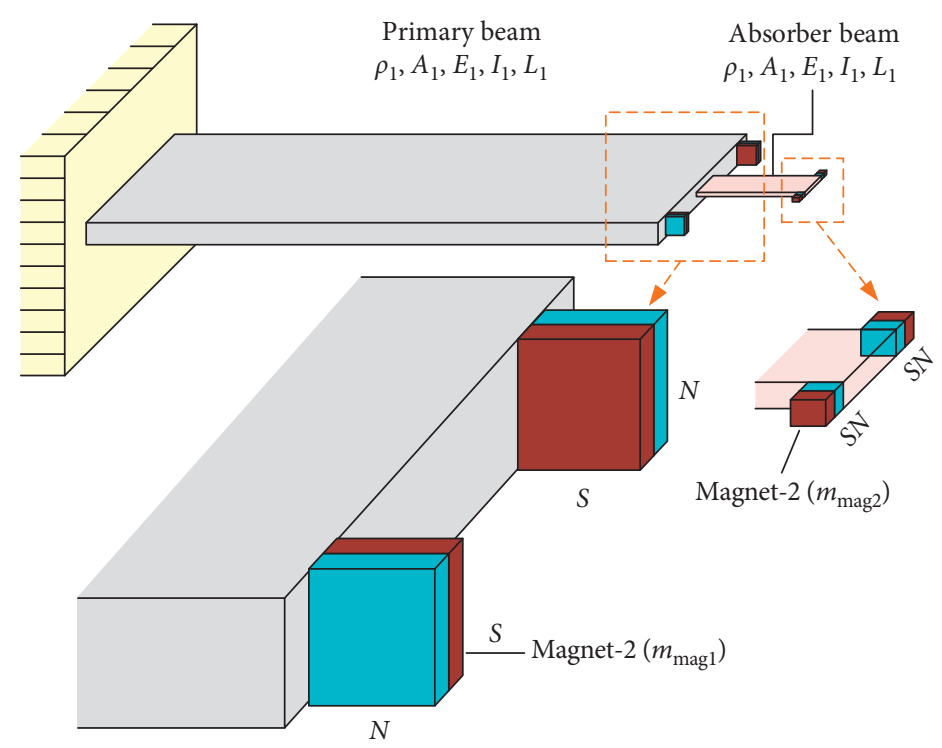

(a)

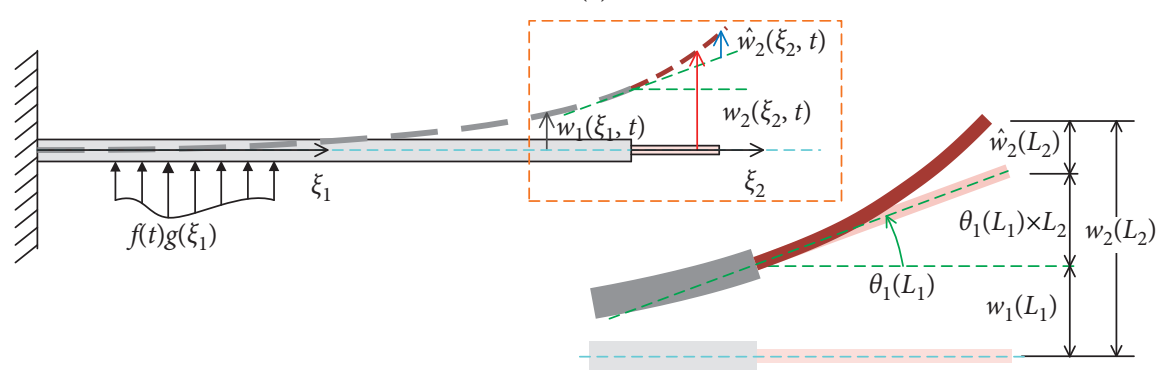

(b)

Figure 1: (a) The model of primary beam and absorber beam coupling with constraint and magnetic interaction; (b) the deformations of system.

TABLE 1: Structural parameters of primary beam and absorber beam.

\begin{tabular}{lc}
\hline Structural parameter & Value (unit) \\
\hline$\rho_{1}, \rho_{2}$ & $1200 \mathrm{~kg} \cdot \mathrm{m}^{3}$ \\
$A_{1}$ & $0.03 \mathrm{~m} \times 0.002 \mathrm{~m}=60 \times 10^{-6} \mathrm{~m}^{2}$ \\
$L_{1}$ & $0.3 \mathrm{~m}$ \\
$I_{1}$ & $20 \times 10^{-12} \mathrm{~mm}^{4}$ \\
$c_{1}$ & $0.1 \mathrm{~N} \cdot \mathrm{s} \cdot \mathrm{m}^{-1}$ \\
$E_{1}, E_{2}$ & $3 \times 10^{9} \mathrm{~Pa}$ \\
$A_{2}$ & $0.005 \mathrm{~m} \times 0.0006 \mathrm{~m}=3 \times 10^{-6} \mathrm{~m}^{2}$ \\
$L_{2}$ & $0.05 \mathrm{~m}$ \\
$I_{2}$ & $0.4167 \times 10^{-12} \mathrm{~mm}^{4}$ \\
$c_{2}$ & $0.1 \mathrm{~N} \cdot \mathrm{s} \cdot \mathrm{m}^{-1}$ \\
\hline
\end{tabular}

2.2. Magnetic Interaction. As shown in Figure 1(a), two pairs of magnets are assembled on the primary and absorber beams. Using one pair of magnets to analyse the interaction force, we obtain the relation between the positions of the two magnets and deflections of the beams. Here, two commoving coordinates are established on the two magnets as $(x, y, z)$ and $\left(x^{\prime}, y^{\prime}, z^{\prime}\right)$, where $x$ and $x^{\prime}$ are coincided with axial direction of beams $\xi_{1}$ and $\xi_{2}$, and $y$ and $y^{\prime}$ are coincided with $w_{1}$ and $w_{2}$. Thus, for vibration deflections $w_{1}$ and $w_{2}$, the locations of the magnet on the primary beam and absorber beam are as shown in Figure 2.
As shown in Figure 2, according to the directions of coordination, the motion $\widehat{w}_{2}\left(L_{2}, t\right)$ occurs in $y$ direction. For the two pairs of magnets, the components of interaction force on magnet- 2 are equilibria in $x$ and $z$ direction, while the components of interaction forces in $y$ direction are not equilibria. The interaction force of one pair of magnets in the deflection direction is in the same direction of $y$, which is written as $F_{\text {mag- } y}$, expressed as

$$
\begin{aligned}
F_{\text {mag-y }}= & \frac{J J^{\prime}}{4 \pi \mu_{0}} \sum_{i=0}^{1} \sum_{j=0}^{1} \sum_{k=0}^{1} \sum_{l=0}^{1} \sum_{p=0}^{1} \sum_{q=0}^{1}(-1)^{i+j+k+l+p+q} \psi_{y} \\
& \cdot\left(u_{i j}, v_{k l}, w_{p q}, r\right),
\end{aligned}
$$

where $\psi_{y}(u, v, w, r)=1 / 2\left(u^{2}-w^{2}\right) \ln (r-v)+u v \ln (r-u)+$ $u w \arctan (u v / r w)+(r v / 2)$; the symbols $u_{i j}, v_{k l}, w_{p q}$, and $r$ are written as

$$
\begin{aligned}
u_{i j} & =L_{2}-A-a+(-1)^{j} A-(-1)^{i} a, \\
v_{k l} & =\left.\widehat{w}_{2}\right|_{L_{2}}+(-1)^{l} B-(-1)^{k} b, \\
w_{p q} & =d-C-c+(-1)^{q} C-(-1)^{p} c, \\
r & =\sqrt{u_{i j}^{2}+v_{k l}^{2}+w_{p q}^{2}} .
\end{aligned}
$$




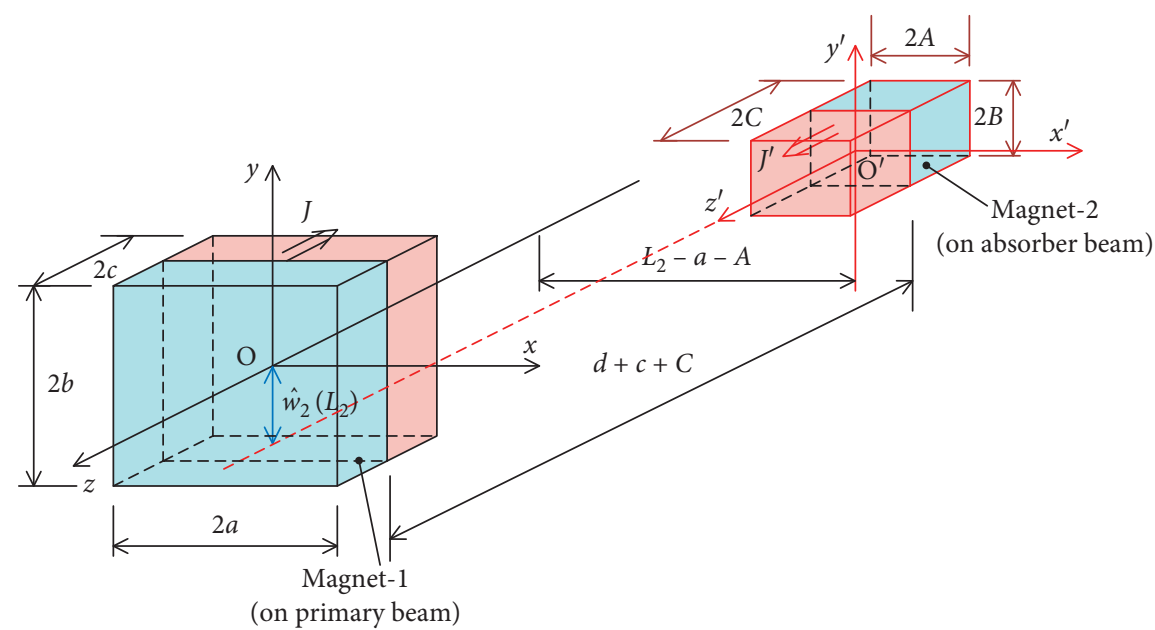

Figure 2: The position of one pair of magnets for deflection.

According to the property of interaction of magnets in $[31,32]$, the interaction force $F_{\text {mag-y }}$ in equation (1) is a complex function with more than 200 terms. To simplify the magnetic interaction force $F_{\text {mag-y }}$ for theoretical analysis and structural design, we utilize high-order polynomial function $F_{y}$ (rather than the Taylor series expansion) to fit the original expression, which is written as

$$
F_{y}=\sum_{h=1}^{H} \alpha_{2 h-1}\left(\left.\widehat{w}_{2}\right|_{L_{2}}\right)^{2 h-1}
$$

where $\alpha_{2 h-1}(h=1,2,3, \ldots, H)$ are fitting coefficients and $H$ reflects the fitting order with sufficient accuracy. Utilizing the Least Square Method (LSM) to find the values of coefficients $\alpha_{2 h-1}(h=1,2,3, \ldots, H)$, the error between the original function $F_{\text {mag- } y}$ and fitting polynomial function $F_{y}$ is defined as

$$
\mathrm{ER}=\frac{\sum_{1}^{H}\left[\left(F_{\text {mag-y }}-F_{y}\right)\left|\widehat{w}_{2}\right|_{L_{2}=w_{i}}\right]^{2}}{H \sum_{1}^{H}\left(F_{\text {mag-y }}\left|\widehat{w}_{2}\right|_{L_{2}=w_{i}}\right)^{2}} .
$$

For different structural parameters $d$, the magnetic interaction force $F_{\text {mag- } y}$ for one pair of magnets, the comparison between the original function $F_{\text {mag- } y}$ and fitting polynomial $F_{y}$, and the relative error ER for various fitting orders $H$ are shown in Figure 3.

Figure 3 shows the variation of one-pair-magnetic interaction force on magnet- 2 in $y$ direction for different structural parameter and the accuracy of the simplification. From the results shown in Figures 3(a) and 3(b), the interaction force varies nonlinearly around the motion equilibrium, more obvious for smaller distance $d$. The gradient of the interaction force at equilibrium is negative, which demonstrates that the magnetic interaction can induce adjustable negative-stiffness property. For the increase of distance $d$ (weaker magnetic field), the gradient becomes negative first and then tends to zero. Thus, the interaction induced by magnetic coupling occurs locally around the equilibrium with adjustable negative-stiffness property and nonlinearity. From the comparison of $F_{y}$ and $F_{\text {mag- } y}$ in Figures 3(b) and 3(c), the fitting polynomial function $F_{y}$ can describe the original force function $F_{\text {mag- } y}$ with high accuracy for $H>7$. Therefore, in the following analysis, to obtain the theoretical solution of the system and propose the design approach, the magnetic interaction force function can be expressed by high-order nonlinear function $F_{y}$.

\section{Model, Analysis, and Structural Design Criterion}

3.1. Vibration Model. Separating the primary and absorber beams and analysing the forces applied on them as shown in Figure 4, the dynamical model can be obtained.

As shown in Figure 4, for the primary beam, it is a cantilever beam which is fixed on the base; for the absorber beam, it is also a cantilever beam fixed on the free end of the primary beam, which is a vibration point rather than a fixed point. According to D'Alembert's principle of a small segment from the primary beam, the dynamical equation of the primary beam is written as

$$
V_{1}+\frac{\partial V_{1}}{\partial \xi_{1}} \mathrm{~d} \xi_{1}-V_{1}-\left(\rho_{1} A_{1} \frac{\partial^{2} w_{1}}{\partial t^{2}}+c_{1} \frac{\partial w_{1}}{\partial t}\right) \mathrm{d} \xi_{1}=f(t) g\left(\xi_{1}\right)
$$

while, for the absorber beam, the dynamical equation of small segment is written as

$$
V_{2}+\frac{\partial V_{2}}{\partial \xi_{2}} \mathrm{~d} \xi_{2}-V_{2}-\left(\rho_{2} A_{2} \frac{\partial^{2} w_{2}}{\partial t^{2}}+c_{2} \frac{\partial w_{2}}{\partial t}\right) \mathrm{d} \xi_{2}=0
$$

The shear force and the bending moment on each section have the general relation as $V=(\partial M / \partial \xi)=$ $(\partial / \partial \xi)\left(-E I\left(\partial^{2} w / \partial \xi^{2}\right)\right)$. Substituting this relation and equation (1) into the dynamical equation of absorber beam and rearranging the two equations, the model is written as 


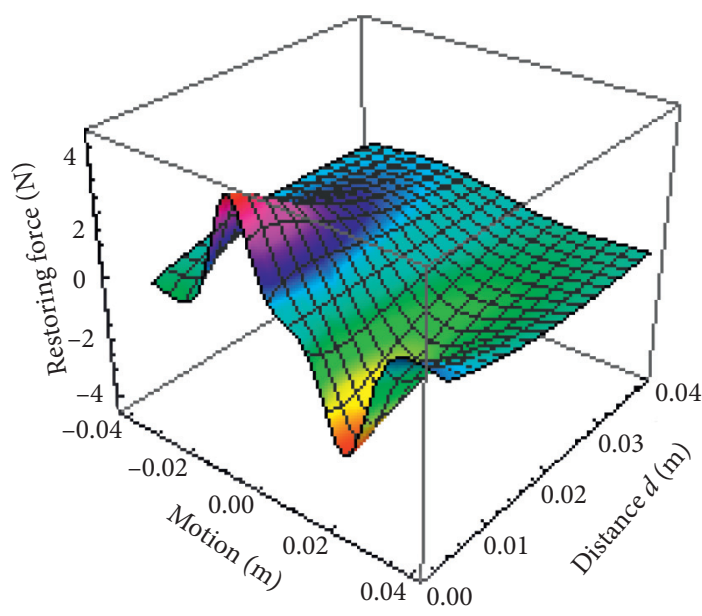

(a)

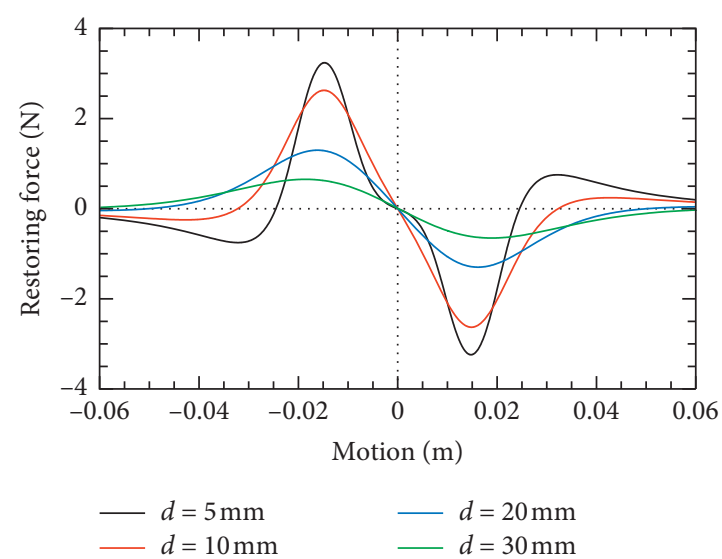

(b)

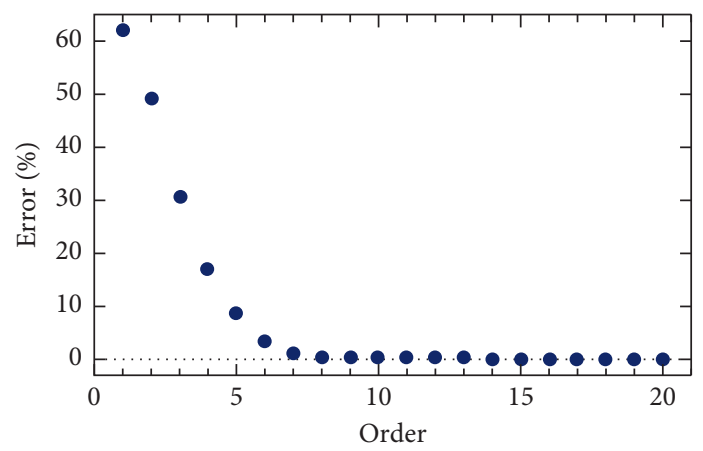

(c)

Figure 3: (a) Magnetic interaction force $F_{\text {mag-y }}$ for different distances $d$; (b) the comparison of the original interaction force $F_{\text {mag- } y}$ (lines) and fitting polynomial function $F_{y}$ (dots) for $H=25$ for $d=5 \mathrm{~mm}, d=10 \mathrm{~mm}, d=20 \mathrm{~mm}$, and $d=30 \mathrm{~mm}$; (c) the error ER between $F_{\text {mag- } y}$ and $F_{y}$ for different fitting orders $N$.

$$
\left\{\begin{array}{l}
\rho_{1} A_{1} \frac{\partial^{2} w_{1}\left(\xi_{1}, t\right)}{\partial t^{2}}+E_{1} I_{1} \frac{\partial^{4} w_{1}\left(\xi_{1}, t\right)}{\partial \xi_{1}^{4}}+c_{1} \frac{\partial w_{1}\left(\xi_{1}, t\right)}{\partial t}=f(t) g\left(\xi_{1}\right) \\
\rho_{2} A_{2} \frac{\partial^{2} \widehat{w}_{2}\left(\xi_{2}, t\right)}{\partial t^{2}}+E_{2} I_{2} \frac{\partial^{4} \widehat{w}_{2}\left(\xi_{2}, t\right)}{\partial \xi_{2}^{4}}+c_{2} \frac{\partial \widehat{w}_{2}\left(\xi_{2}, t\right)}{\partial t}=-c_{2}\left(\frac{\mathrm{d} w_{1}\left(L_{1}, t\right)}{\mathrm{d} t}+\frac{\mathrm{d} \theta_{1}\left(L_{1}, t\right)}{\mathrm{d} t}\right)-\rho_{2} A_{2}\left[\frac{\mathrm{d}^{2} w_{1}\left(L_{1}, t\right)}{\mathrm{d} t^{2}}+\frac{\mathrm{d}^{2} \theta_{1}\left(L_{1}, t\right)}{\mathrm{d} t^{2}}\right] \xi_{2} .
\end{array}\right.
$$

According to the forces analysis on the beams shown in Figure 4, at the free end of the primary beam, the interaction force of magnets $F_{y}$ is applied on magnet-1; similarly, for the absorber beam, the interaction force of magnet $F_{y}$ is applied on magnet-2 at its free end. At the connection section $\xi_{1}=L_{1}\left(\xi_{2}=0\right)$, the forces $V_{1}\left(L_{1}\right)$ and
$V_{2}^{\prime}(0)$ are one pair of reaction forces, which are equal and in the opposite direction, as similarly as the bending moments $M_{1}\left(L_{1}\right)$ and $M_{2}^{\prime}(0)$. Thus, we can obtain the boundary conditions at $\xi_{1}=0$ and $\xi_{2}=L_{2}$, respectively, and the compatibility conditions of the connection section at $\xi_{1}=L_{1}\left(\xi_{2}=0\right)$ as 


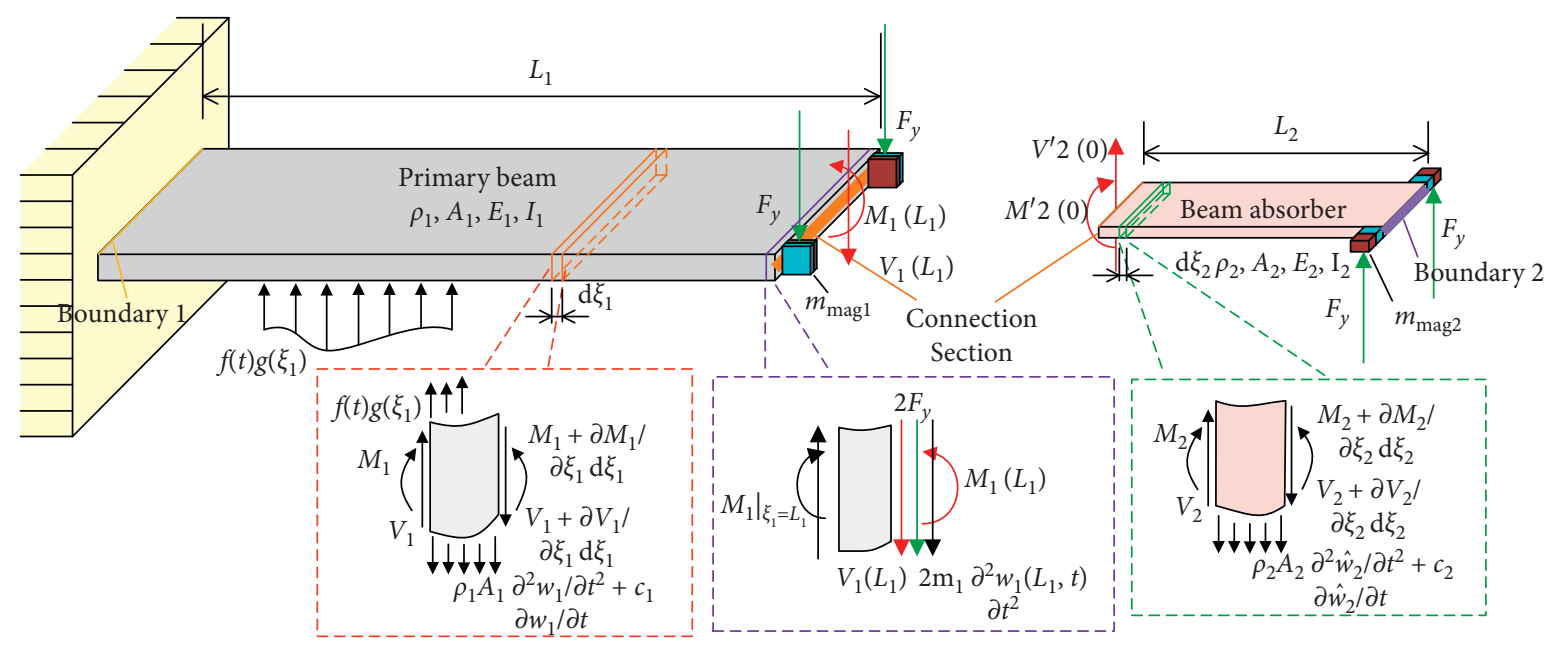

FIgURE 4: The forces applied on segment, boundary, and compatibility at connection section.

$$
\left\{\begin{array}{l}
\left.w_{1}\left(\xi_{1}, t\right)\right|_{\xi_{1}=0}=0, \\
\left.w_{1}^{\prime}\left(\xi_{1}, t\right)\right|_{\xi_{1}=0}=0, \\
\left.E_{2} I_{2} w_{2}^{\prime \prime \prime}\left(\xi_{2}, t\right)\right|_{\xi_{2}=L_{2}}=\left.2 m_{\mathrm{mag} 2} \ddot{w}_{2}\left(\xi_{2}, t\right)\right|_{\xi_{2}=L_{2}}-2 F_{y}\left(\widehat{w}_{2}\left(L_{2}, t\right)\right),\left.E_{2} I_{2} w_{2}^{\prime \prime}\left(\xi_{2}, t\right)\right|_{\xi_{2}=L_{2}}=0, \\
\left.w_{1}\left(\xi_{1}, t\right)\right|_{\xi_{1}=L_{1}}=\left.w_{2}\left(\xi_{2}, t\right)\right|_{\xi_{2}=0},\left.w_{1}^{\prime}\left(\xi_{1}, t\right)\right|_{\xi_{1}=L_{1}}=\left.w_{2}^{\prime}\left(\xi_{2}, t\right)\right|_{\xi_{2}=0}, \\
\left.E_{1} I_{1} w_{1}^{\prime \prime \prime}\left(\xi_{1}, t\right)\right|_{\xi_{1}=L_{1}}-\left.2 m_{\mathrm{mag} 1} \ddot{w}_{1}\left(\xi_{1}, t\right)\right|_{\xi_{1}=L_{1}}-2 F_{y}\left(\widehat{w}_{2}\left(L_{2}, t\right)\right)=\left.E_{2} I_{2} w_{2}^{\prime \prime \prime}\left(\xi_{2}, t\right)\right|_{\xi_{2}=0}, \\
\left.E_{1} I_{1} w_{1}^{\prime \prime}\left(\xi_{1}, t\right)\right|_{\xi_{1}=L_{1}}=\left.E_{2} I_{2} w_{2}^{\prime \prime}\left(\xi_{2}, t\right)\right|_{\xi_{2}=0} .
\end{array}\right.
$$

Since the primary beam and absorber beam are both cantilever beams, the mode shape functions $\phi_{1 i}$ and $\phi_{2 i}$ are set as

$$
\begin{aligned}
& \phi_{1 i}\left(\xi_{1}\right)=B_{1 i}\left\{\cos \left(\frac{\lambda_{i} \xi_{1}}{L_{1}}\right)-\cosh \left(\frac{\lambda_{i} \xi_{1}}{L_{1}}\right)+r_{1 i}\left[\sin \left(\frac{\lambda_{i} \xi_{1}}{L_{1}}\right)-\sinh \left(\frac{\lambda_{i} \xi_{1}}{L_{1}}\right)\right]\right\}, \\
& \phi_{2 j}\left(\xi_{2}\right)=B_{2 j}\left\{\cos \left(\frac{\lambda_{j} \xi_{2}}{L_{2}}\right)-\cosh \left(\frac{\lambda_{j} \xi_{2}}{L_{2}}\right)+r_{2 j}\left[\sin \left(\lambda_{j} \xi_{2} / L_{2}\right)-\sinh \left(\frac{\lambda_{j} \xi_{2}}{L_{2}}\right)\right]\right\},
\end{aligned}
$$

where $\quad r_{1 i}=-\left(\cos \lambda_{i}+\cosh \lambda_{i}\right) /\left(\sin \lambda_{i}+\sinh \lambda_{i}\right) \quad$ and $r_{2 j}=-\left(\cos \lambda_{j}+\cosh \lambda_{j}\right) /\left(\sin \lambda_{j}+\sinh \lambda_{j}\right)$. The functions $\phi_{1 i}$ and $\phi_{2 j}$ should satisfy the orthogonality and normalization principle, written as

$$
\begin{aligned}
& \int_{0}^{L_{1}} \rho_{1} A_{1} \phi_{1 i}\left(\xi_{1}\right) \phi_{1 k}\left(\xi_{1}\right) \mathrm{d} \xi_{1}=\delta_{i k}=\left\{\begin{array}{ll}
1, & i=k, \\
0, & i \neq k,
\end{array} \quad i, k=1,2,3, \ldots,\right. \\
& \int_{0}^{L_{2}} \rho_{2} A_{2} \phi_{2 j}\left(\xi_{2}\right) \phi_{2 n}\left(\xi_{2}\right) \mathrm{d} \xi_{2}=\delta_{j n}=\left\{\begin{array}{ll}
1, & j=n, \\
0, & j \neq n,
\end{array} \quad j, n=1,2,3, \ldots,\right. \\
& E_{1} I_{1} \int_{0}^{L_{1}} \phi_{1 i}^{\prime \prime}\left(\xi_{1}\right) \phi_{1 k}^{\prime \prime}\left(\xi_{1}\right) \mathrm{d} \xi_{1}=\Omega_{1 k}^{2} \delta_{i k}, \\
& E_{2} I_{2} \int_{0}^{L_{2}} \phi_{2 j}^{\prime \prime}\left(\xi_{2}\right) \phi_{2 n}^{\prime \prime}\left(\xi_{2}\right) \mathrm{d} \xi_{2}=\omega_{2 n}^{2} \delta_{j n} .
\end{aligned}
$$


Truncating the vibration responses of primary system for $K^{\text {th }}$ order and absorber beam for $N^{\text {th }}$ order as

$$
\begin{aligned}
& w_{1}\left(\xi_{1}, t\right)=\sum_{i=1}^{K} \phi_{1 i}\left(\xi_{1}\right) q_{1 i}(t), \\
& \widehat{w}_{2}\left(\xi_{2}, t\right)=\sum_{j=1}^{N} \phi_{2 j}\left(\xi_{2}\right) q_{2 j}(t),
\end{aligned}
$$

and substituting equation (9) into the dynamical equation (7) and applying partial integration, the following equations can be obtained:

$$
\begin{aligned}
& \left.E_{1} I_{1} w_{1}^{\prime \prime \prime}\left(\xi_{1}, t\right) \phi_{1 k}\left(\xi_{1}\right)\right|_{0} ^{L_{1}}-\left.E_{1} I_{1} w_{1}^{\prime \prime}\left(\xi_{1}, t\right) \phi_{1 k}^{\prime}\left(\xi_{1}\right)\right|_{0} ^{L_{1}}+E_{1} I_{1} \sum_{i} \int_{0}^{L_{1}} \phi_{1 i}^{\prime \prime}\left(\xi_{1}\right) \phi_{1 k}^{\prime \prime}\left(\xi_{1}\right) \mathrm{d} \xi_{1} q_{1 i}(t) \\
& \quad+\rho_{1} A_{1} \sum_{i} \int_{0}^{L_{1}} \phi_{1 i}\left(\xi_{1}\right) \phi_{1 k}\left(\xi_{1}\right) \mathrm{d} \xi_{1} \ddot{q}_{1 i}(t)+c_{1} \sum_{i} \int_{0}^{L_{1}} \phi_{1 i}\left(\xi_{1}\right) \phi_{1 k}\left(\xi_{1}\right) \mathrm{d} \xi_{1} \dot{q}_{1 i}(t) \\
& =\int_{0}^{L_{1}} f(t) \delta\left(\xi_{1}-l_{1}\right) \phi_{1 k}\left(\xi_{1}\right) \mathrm{d} \xi_{1}, \\
& \left.E_{2} I_{2} \widehat{w}_{2}^{\prime \prime \prime}\left(\xi_{2}, t\right) \phi_{2 n}\left(\xi_{2}\right)\right|_{0} ^{L_{2}}-\left.E_{2} I_{2} \widehat{w}_{2}^{\prime \prime}\left(\xi_{2}, t\right) \phi_{2 n}^{\prime}\left(\xi_{2}\right)\right|_{0} ^{L_{2}}+E_{2} I_{2} \sum_{j} \int_{0}^{L_{2}} \phi_{2 j}^{\prime \prime}\left(\xi_{2}\right) \phi_{2 n}^{\prime \prime}\left(\xi_{2}\right) \mathrm{d} \xi_{2} q_{2 j}(t) \\
& \quad+\rho_{2} A_{2} \sum_{j} \int_{0}^{L_{2}} \phi_{2 j}\left(\xi_{2}\right) \phi_{2 n}\left(\xi_{2}\right) \mathrm{d} \xi_{2} \ddot{q}_{2 j}(t)+c_{2} \sum_{j} \int_{0}^{L_{2}} \phi_{2 j}\left(\xi_{2}\right) \phi_{2 n}\left(\xi_{2}\right) \mathrm{d} \xi_{2} \dot{q}_{2 j}(t) \\
& =-c_{2} \sum_{i} \int_{0}^{L_{2}}\left[\phi_{1 i}^{\prime}\left(L_{1}\right)+\phi_{1 i}\left(L_{1}\right)\right] \phi_{2 n}\left(\xi_{2}\right) \mathrm{d} \xi_{2} \dot{q}_{1 i}(t)-\rho_{2} A_{2} \sum_{i} \int_{0}^{L_{2}}\left[\phi_{1 i}^{\prime}\left(L_{1}\right)+\phi_{1 i}\left(L_{1}\right)\right] \phi_{2 n}\left(\xi_{2}\right) \xi_{2} \mathrm{~d} \xi_{2} \ddot{q}_{1 i}(t) .
\end{aligned}
$$

In equation (12), by considering the boundary conditions and compatibility conditions as equation (8), the terms by definite integration are

$$
\begin{aligned}
\left.E_{1} I_{1} w_{1}^{\prime \prime \prime}\left(\xi_{1}, t\right) \phi_{1 k}\left(\xi_{1}\right)\right|_{0} ^{L_{1}} & =\left.E_{1} I_{1} w_{1}^{\prime \prime \prime}\left(\xi_{1}, t\right)\right|_{\xi_{1}=L_{1}} \phi_{1 k}\left(L_{1}\right)-\left.E_{1} I_{1} w_{1}^{\prime \prime \prime}\left(\xi_{1}, t\right)\right|_{\xi_{1}=0} \phi_{1 k}(0) \\
& =\left[\left.E_{2} I_{2} \widehat{w}_{2}^{\prime \prime \prime}\left(\xi_{2}, t\right)\right|_{\xi_{2}=0}+\left.2 m_{\text {mag1 }} \ddot{w}_{1}\left(\xi_{1}, t\right)\right|_{\xi_{1}=L_{1}}+2 F_{y}\left(\widehat{w}_{2}\left(L_{2}, t\right)\right)\right] \phi_{1 k}\left(L_{1}\right), \\
\left.E_{1} I_{1} w_{1}^{\prime \prime}\left(\xi_{1}, t\right) \phi_{1 k}^{\prime}\left(\xi_{1}\right)\right|_{0} ^{L_{1}} & =\left.E_{1} I_{1} w_{1}^{\prime \prime}\left(\xi_{1}, t\right)\right|_{\xi_{1}=L_{1}} \phi_{1 k}^{\prime}\left(L_{1}\right)-\left.E_{1} I_{1} w_{1}^{\prime \prime}\left(\xi_{1}, t\right)\right|_{\xi_{1}=0} \phi_{1 k}^{\prime}(0) \\
& =\left.E_{2} I_{2} \widehat{w}_{2}^{\prime \prime}\left(\xi_{2}, t\right)\right|_{\xi_{2}=0} \phi_{1 k}^{\prime}\left(L_{1}\right), \\
\left.E_{2} I_{2} \widehat{w}_{2}^{\prime \prime \prime}\left(\xi_{2}, t\right) \phi_{2 n}\left(\xi_{2}\right)\right|_{0} ^{L_{2}} & =\left.E_{2} I_{2} \widehat{w}_{2}^{\prime \prime \prime}\left(\xi_{2}, t\right)\right|_{\xi_{2}=L_{2}} \phi_{2 n}\left(L_{2}\right)-\left.E_{2} I_{2} \widehat{w}_{2}^{\prime \prime \prime}\left(\xi_{2}, t\right)\right|_{\xi_{2}=0} \phi_{2 n}(0) \\
& =2 m_{\text {mag2 } 2}\left[\left.\ddot{\widehat{w}}_{2}\left(\xi_{2}, t\right)\right|_{\xi_{2}=L_{2}}+\ddot{\theta}_{1}\left(L_{1}, t\right) L_{2}+\ddot{w}_{1}\left(L_{1}, t\right)\right] \phi_{2 n}\left(L_{2}\right)-2 F_{y}\left(\widehat{w}_{2}\left(L_{2}, t\right)\right) \phi_{2 n}\left(L_{2}\right), \\
\left.E_{2} I_{2} \widehat{w}_{2}^{\prime \prime}\left(\xi_{2}, t\right) \phi_{2 n}^{\prime}\left(\xi_{2}\right)\right|_{0} ^{L_{2}} & =\left.E_{2} I_{2} \widehat{w}_{2}^{\prime \prime}\left(\xi_{2}, t\right)\right|_{\xi_{2}=L_{2}} \phi_{2 n}^{\prime}\left(L_{2}\right)-\left.E_{2} I_{2} \widehat{w}_{2}^{\prime \prime}\left(\xi_{2}, t\right)\right|_{\xi_{2}=0} \phi_{2 n}^{\prime}(0)=0 .
\end{aligned}
$$


After simplification, the discretization equation of the system can be written as

$$
\begin{aligned}
& \ddot{q}_{1 k}(t)+\Omega_{1 k}^{2} q_{1 k}(t)+C_{1 k} \dot{q}_{1 k}(t)
\end{aligned}
$$

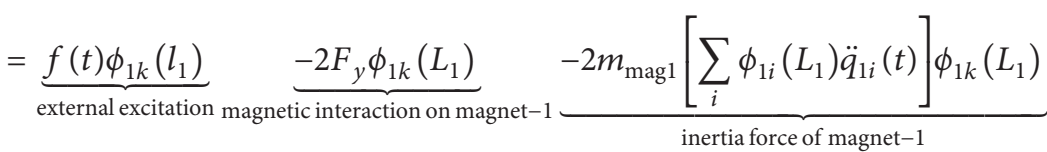

$$
\begin{aligned}
& \underbrace{-E_{2} I_{2}\left[\sum_{j} \phi_{2 j}^{\prime \prime \prime}(0) q_{2 j}(t)\right] \phi_{1 k}\left(L_{1}\right)}_{\text {coupling shear force induced by absorber }} \underbrace{+E_{2} I_{2}\left[\sum_{j} \phi_{2 j}^{\prime \prime \prime}(0) q_{2 j}(t)\right] \phi_{1 k}^{\prime}\left(L_{1}\right)}_{\text {coupling bending moment induced by absorber }}, \\
& \ddot{q}_{2 n}(t)+\omega_{2 n}^{2} q_{2 n}(t)+C_{2 n} \dot{q}_{2 n}(t) \\
& =\underbrace{+2 F_{y} \phi_{2 n}\left(L_{2}\right)}_{\text {magnetic interaction on magnet }-2} \underbrace{-C_{3 n} \sum_{i} \phi_{1 i}^{\prime}\left(L_{1}\right) \dot{q}_{1 i}(t)-m_{c n} \sum_{i} \phi_{1 i}^{\prime}\left(L_{1}\right) \ddot{q}_{1 i}(t)}_{\text {effect of rotation at free end of primary beam }} \\
& -C_{3 n} \sum_{i} \phi_{1 i}\left(L_{1}\right) \dot{q}_{1 i}(t)-m_{c n} \sum_{i} \phi_{1 i}\left(L_{1}\right) \ddot{q}_{1 i}(t) \\
& -2 m_{\mathrm{mag} 2}\left[\sum_{j} \phi_{2 j}\left(L_{2}\right) \ddot{q}_{2 j}(t)+\sum_{i} \phi_{1 i}^{\prime}\left(L_{1}\right) \ddot{q}_{1 i}(t) L_{2}+\sum_{i} \phi_{1 i}\left(L_{1}\right) \ddot{q}_{1 i}(t)\right] \phi_{2 n}\left(L_{2}\right), \\
& \text { ineria force of magnet-2 }
\end{aligned}
$$

where

$$
\begin{aligned}
& C_{1 k}=c_{1} \int_{0}^{L_{1}} \phi_{1 i}\left(\xi_{1}\right) \phi_{1 k}\left(\xi_{1}\right) \mathrm{d} \xi_{1}=c_{1} \delta_{i k}, \\
& C_{2 n}=c_{2} \int_{0}^{L_{2}} \phi_{2 j}\left(\xi_{1}\right) \phi_{2 n}\left(\xi_{2}\right) \mathrm{d} \xi_{2}=c_{2} \delta_{j n}, \\
& C_{3 n}=c_{2} \int_{0}^{L_{2}} \phi_{2 n}\left(\xi_{2}\right) \mathrm{d} \xi_{2}, \\
& m_{c n}=\rho_{2} A_{2} \int_{0}^{L_{2}} \phi_{2 n}\left(\xi_{2}\right) \xi_{2} \mathrm{~d} \xi_{2} .
\end{aligned}
$$

According to equation (14) and the definitions of coefficients (15), for $K^{\text {th }}$ order and $N^{\text {th }}$ order truncation for the primary beam and absorber beam, respectively, the discretization equation is written as

$$
\mathbf{M} \ddot{q}(t)+\mathbf{K q}(t)+\mathbf{C} \dot{q}(t)=\mathbf{F}(t)+\mathbf{G}(q),
$$

where $\mathbf{M}$ is the mass matrix, $\mathbf{K}$ is the linear stiffness matrix, $\mathbf{C}$ is the linear damping matrix, $\mathbf{F}$ is the excitation vector, and $\mathbf{G}$ is the vector for nonlinear terms, expressed as follows. The mass matrix is

$$
\begin{aligned}
\mathbf{M} & =\left[\begin{array}{ll}
\mathbf{M}_{i k} & \mathbf{0} \\
\mathbf{M}_{j i} & \mathbf{M}_{j n}
\end{array}\right], \\
m_{i k} & = \begin{cases}1+2 m_{\mathrm{mag} 1} \phi_{1 i}\left(L_{1}\right) \phi_{1 k}\left(L_{1}\right), & i=k, \\
2 m_{\mathrm{mag} 1} \phi_{1 i}\left(L_{1}\right) \phi_{1 k}\left(L_{1}\right), & i \neq k,\end{cases} \\
m_{j i} & =2 m_{\mathrm{mag} 2}\left[\phi_{1 i}^{\prime}\left(L_{1}\right) L_{2}+\phi_{1 i}\left(L_{1}\right)\right] \phi_{2 j}\left(L_{2}\right)+m_{c n} \phi_{1 i}\left(L_{1}\right), \\
m_{j n} & = \begin{cases}1+2 m_{\mathrm{mag} 2} \phi_{2 j}\left(L_{2}\right) \phi_{2 n}\left(L_{2}\right), & j=n, \\
2 m_{\mathrm{mag} 2} \phi_{2 j}\left(L_{2}\right) \phi_{2 n}\left(L_{2}\right), & j \neq n .\end{cases}
\end{aligned}
$$

The expression of $\mathbf{K}$ is 


$$
\begin{aligned}
\mathbf{K} & =\left[\begin{array}{cc}
\mathbf{K}_{i k} & \mathbf{K}_{k j} \\
\mathbf{0} & \mathbf{K}_{j n}
\end{array}\right], \\
k_{i k} & =\left\{\begin{array}{ll}
\Omega_{1 k}^{2} & i=k \\
0 & i \neq k
\end{array},\right. \\
k_{k j} & =2 \alpha_{1} \phi_{2 j}\left(L_{1}\right) \phi_{1 k}\left(L_{1}\right)+E_{2} I_{2} \phi_{2 j}^{\prime \prime \prime}(0) \phi_{1 k}\left(L_{1}\right)-E_{2} I_{2} \phi_{2 j}^{\prime \prime}(0) \phi_{1 k}^{\prime}\left(L_{1}\right), \\
k_{j n} & = \begin{cases}\omega_{2 n}^{2}+2 m_{\text {mag } 2} \phi_{2 j}^{\prime \prime}\left(L_{2}\right) \phi_{2 n}\left(L_{2}\right)-2 \alpha_{1} \phi_{2 j}\left(L_{2}\right) \phi_{2 n}\left(L_{2}\right) & j=n, \\
2 m_{\text {mag } 2} \phi_{2 j}^{\prime \prime}\left(L_{2}\right) \phi_{2 n}\left(L_{2}\right)-2 \alpha_{1} \phi_{2 j}\left(L_{2}\right) \phi_{2 n}\left(L_{2}\right) & j \neq n,\end{cases}
\end{aligned}
$$

The expression of $\mathbf{C}$ is

$$
\begin{aligned}
\mathbf{C}= & {\left[\begin{array}{ll}
\mathbf{C}_{i k} & 0 \\
\mathbf{C}_{i n} & \mathbf{C}_{j n}
\end{array}\right], } \\
c_{i k}= & \begin{cases}c_{1}, & i=k, \\
0, & i \neq k,\end{cases} \\
c_{i n}= & C_{3 n}\left[\phi_{1 k}^{\prime}\left(L_{1}\right)+\phi_{1 k}\left(L_{1}\right)\right]=c_{2} \int_{0}^{L_{2}} \phi_{2 n}\left(\xi_{2}\right) \mathrm{d} \xi_{2}\left[\phi_{1 k}^{\prime}\left(L_{1}\right)\right. \\
& \left.+\phi_{1 k}\left(L_{1}\right)\right], \\
c_{j n}= & \begin{cases}c_{2}, & j=n, \\
0, & j \neq n .\end{cases}
\end{aligned}
$$

The nonlinearity terms vector $\mathbf{G}$ is written as

$$
\begin{aligned}
G & =\left\{g_{11}, g_{12}, \ldots, g_{1 K}, g_{21}, g_{22}, \ldots, g_{2 N}\right\}^{T}, \\
g_{1 k} & =-2 F_{y} \phi_{1 k}\left(L_{1}\right)=-2 \sum_{h=1}^{H}\left[\alpha_{2 h-1}\left(\sum_{j=1}^{N} \phi_{2 j}\left(L_{2}\right) q_{2 j}(t)\right)^{2 h-1}\right] \phi_{1 k}\left(L_{1}\right), \\
g_{2 n} & =2 F_{y} \phi_{2 n}\left(L_{2}\right)=2 \sum_{h=1}^{H}\left[\alpha_{2 n-1}\left(\sum_{j=1}^{N} \phi_{2 j}\left(L_{2}\right) q_{2 j}(t)\right)^{2 h-1}\right] \phi_{2 n}\left(L_{2}\right) .
\end{aligned}
$$

According to the dynamical model (16), coefficients matrix, and nonlinearity terms in (17)-(20), the natural frequencies and responses can be obtained for different structural parameters under different excitations.

\section{Isolation Effectiveness}

4.1. Resonance Frequency Bands. First, the natural frequencies of the system are obtained by the linearization model of equation (14) to show the resonance frequency bands after the assembly of absorber beam with magnetic interaction. According to the defined matrix $\mathbf{M}$ and $\mathbf{K}$ as (19) and (20), the natural frequencies for linearization can be solved by

$$
\operatorname{det}\left(\mathbf{M} \widetilde{\Omega}_{\beta}^{2}-\mathbf{K}\right)=0,
$$

where $\widetilde{\Omega}_{\beta}(\beta=K+N)$ are the linearization natural frequencies for the system. Then, the comparison of the natural frequencies $\widetilde{\Omega}_{\beta}$ of the system with/without magnetic interaction coupling is shown in Figure 5.
The natural frequencies of the system for different orders of Galerkin truncation are calculated and the relative errors are shown and listed in Table 2 in Appendix. In Table 2, when the truncation order of the absorber beam is $N=3, \widetilde{\Omega}_{10}$ is missed; when the truncation order of the primary beam is $K=4$ or $K=5, \widetilde{\Omega}_{7}$ and $\widetilde{\Omega}_{8}$ would be missed; when the truncation order of the primary beam is set as $K=6$, there are no omissions for natural frequencies in the discussed frequency band as shown in Figure 5. The truncation order of the primary beam is higher than the absorber beam since the primary beam has lower stiffness than the absorber beam.

Figure 5 shows comparison among the cases for primary beam without absorber beam (dot-dashed lines), with absorber beam but without magnetic interaction (dashed lines), and with both absorber beam and magnetic interaction (lines with dots). The two pairs of magnets installed in the system mainly affect the resonance frequency of the first few orders. They do not have much influence on the resonance frequency of higher orders. Figure 5(b) shows the resonance frequency of the first three orders. Without the absorber, there are only two modes in this frequency band, which are plotted as dot-dashed lines. The first natural frequency of the primary system is $27.03 \mathrm{rad} / \mathrm{s}$ and the second one is $169.4 \mathrm{rad} / \mathrm{s}$. When the absorber beam is assembled but without magnetic interaction $\left(F_{y}=0\right)$, the first natural frequency equals $22.1 \mathrm{rad} / \mathrm{s}$, the second one $66.46 \mathrm{rad} / \mathrm{s}$, and the third one $190.6 \mathrm{rad} / \mathrm{s}$. For the case with both absorber beam and magnetic interaction, each order of natural frequencies depends on the interaction field. For increasing the distance $d$ of one pair of magnets, the first natural frequency reduces closely to zero and then increases closer to $22.1 \mathrm{rad} / \mathrm{s}$ since the interaction is weakened for very large distance $d$. Similarly, the second and third natural frequencies increase for the increase of $d$ and then reduce closer to the values of $66.46 \mathrm{rad} / \mathrm{s}$ and $190.6 \mathrm{rad} / \mathrm{s}$.

Since the first frequency of the system is very close to zero and the second one reaches a maximum value for $d \approx 12 \mathrm{~mm}$, there is a wide frequency band from zero for nonresonance where the vibration is suppressed effectively. In the following analysis, the amplitude frequency curves of the primary beam for different structural parameters are solved by perturbation method to show the vibration suppression effectiveness of absorber beam with nonlinear interaction by magnetic field.

The first natural frequency first decreases and then increases. It can decrease to approximately zero. The second 


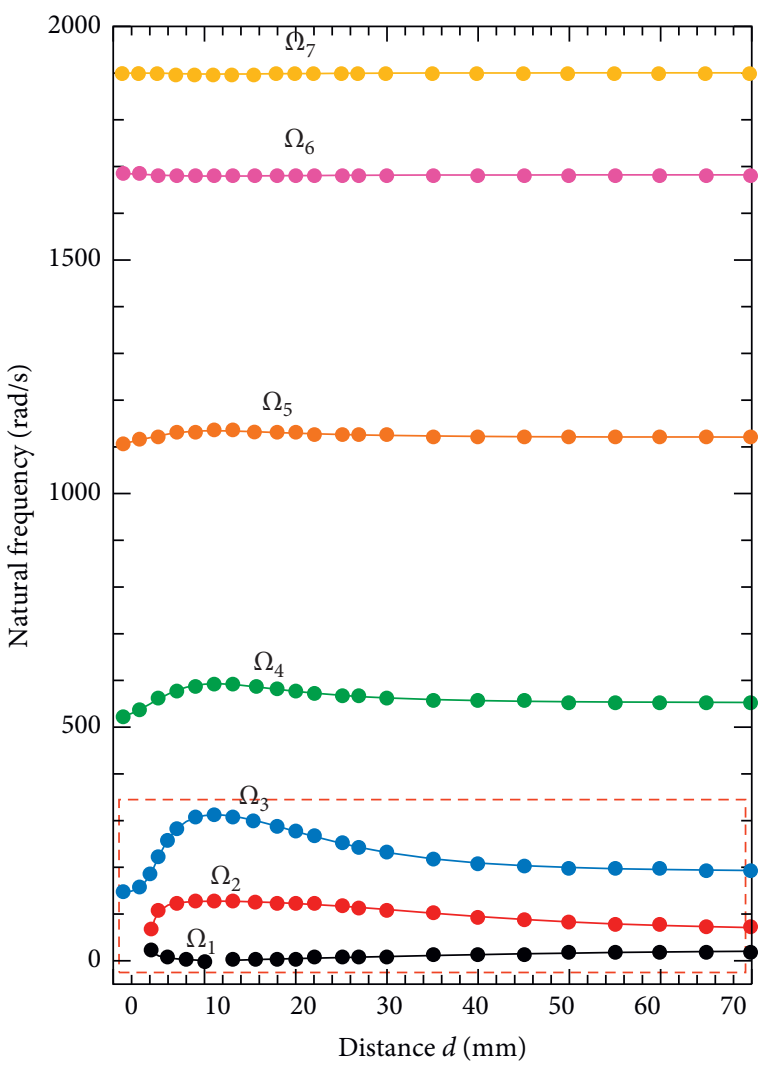

(a)

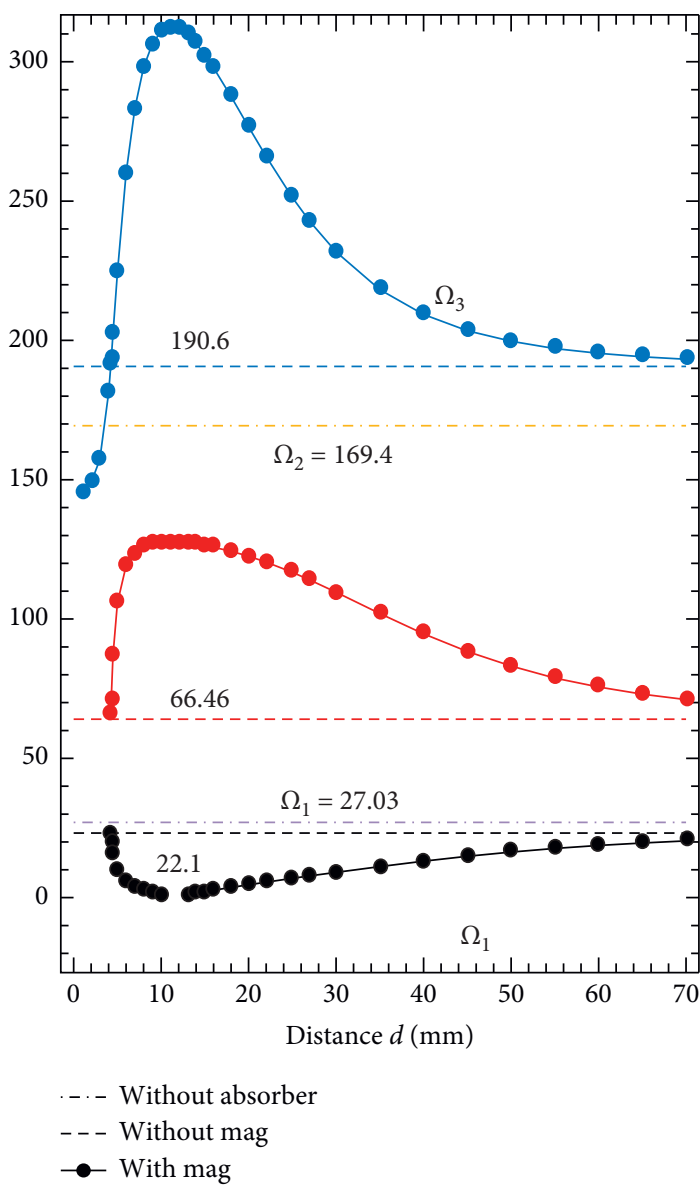

(b)

FiguRE 5: (a) The natural frequencies for different structural parameters $d$; (b) the first three natural frequencies and the comparison cases with/without the absorber beam and with/without the magnetic interaction.

TABle 2: Natural frequencies for different truncations $K$ and $N$ for $\alpha_{1}=-100(d=23 \mathrm{~mm})$.

\begin{tabular}{lcccccc}
\hline$\widetilde{\Omega}_{\beta}(\mathrm{rad} / \mathrm{s})$ & $K=6, N=4$ & $K=6, N=3$ & $K=5, N=4$ & $K=5, N=3$ & $K=4, N=4$ & $K=4, N=3$ \\
\hline$\widetilde{\Omega}_{1}$ & 5.79 & 5.79 & 5.79 & 5.79 & 5.77 \\
$\widetilde{\Omega}_{2}$ & 119.57 & 119.59 & 119.79 & 119.82 & 120.2 \\
$\widetilde{\Omega}_{3}$ & 261.97 & 262.12 & 262.19 & 262.47 & 262.5 & 566.6 \\
$\widetilde{\Omega}_{4}$ & 565.72 & 566.04 & 565.93 & 566.25 & 119.65 \\
$\widetilde{\Omega}_{5}$ & 1110.3 & 1110.87 & 1113.35 & 1113.89 & 177.47 \\
$\widetilde{\Omega}_{6}$ & 1691.28 & 1692.22 & 1698.46 & 1703.2 & 1126.2 \\
$\widetilde{\Omega}_{7}$ & 1904.95 & 1903.98 & 1911.77 & 1910.93 & 1692.1 \\
$\widetilde{\Omega}_{8}$ & 2862.24 & 2861.98 & & & 5547.1 \\
$\widetilde{\Omega}_{9}$ & 5533.08 & 5619.92 & 5540.21 & 5627.01 & 5634.28 \\
$\widetilde{\Omega}_{10}$ & 11718.2 & 11717.9 & 11717.6 & & \\
\hline
\end{tabular}

natural frequency increased to maximum. Therefore, the magnetic interaction further optimized the effective absorption band, allowing it to start from approximately zero. Without the absorber, frequency ranges within $22 \sim 66 \mathrm{rad} / \mathrm{s}$. With the absorber, the frequency range is widened to $0 \sim 150 \mathrm{rad} / \mathrm{s}$. This is the novelty of the structure design. Moreover, there is no need to install or uninstall the magnets while working. If the magnetic interaction is not needed, increasing the magnetic distance $d$ to a certain value can eliminate the influence of the magnets and allow the structure to turn back to its original state. The system can be easily optimized by adjusting $d$ value.

4.2. Solutions on Frequency Band. Based on the discretization equation (16), without considering the internal resonance, the vibration solutions of the primary beam $q_{1 i}(t)$ and the absorber beam $q_{2 i}(t)$ for different orders of modal are written as 


$$
\begin{aligned}
& q_{1 i}(t)=\sum_{m}\left[a_{1 i m} \cos (m \Omega t)+b_{1 i m} \sin (m \Omega t)\right], \\
& q_{2 j}(t)=\sum_{m}\left[a_{2 j m} \cos (m \Omega t)+b_{2 j m} \sin (m \Omega t)\right] .
\end{aligned}
$$

Substituting the solution in (22) into equation (16) and choosing the same order of harmonic components $\cos (m \Omega t)$ and $\sin (m \Omega t)$, we obtain $2 \times m \times \beta=2 \times m \times(K+N)$ algebraic equations for $a_{1 i m}, b_{1 i m}, a_{2 j m}$, and $b_{2 j m}$. In the following analysis of amplitude-frequency curves, single harmonic components for primary resonances are considered and the super/subharmonic components are ignored; then, there are $2 \times \beta$ algebraic equations for solving the $2 \times \beta$ amplitudes.

According to the relation between the vibration response $w_{2}$ and vibration deflection $\widehat{w}_{2}$ in equation (1), the vibration response $w_{2}\left(\xi_{2}, t\right)$ is written as

$$
\begin{aligned}
w_{2}\left(\xi_{2}, t\right)= & \sum_{i=1}^{K} \phi_{1 i}\left(L_{1}\right) q_{1 i}(t)+\sum_{i=1}^{K} \phi_{1 i}^{\prime}\left(L_{1}\right) q_{1 i}(t) \xi_{2} \\
& +\sum_{j=1}^{N} \phi_{2 j}\left(L_{2}\right) q_{2 j}(t) .
\end{aligned}
$$

Thus, the maximum vibration response of the primary beam $w_{1}\left(L_{1}, t\right)$, relative motion $\widehat{w}_{2}\left(L_{2}, t\right)$, and the maximum vibration response of the absorber beam $w_{2}\left(L_{1}, t\right)$ are, respectively, written as

$$
\begin{aligned}
w_{1}\left(L_{1}, t\right) & =\sqrt{\left(\sum_{i} \phi_{1 i}\left(L_{1}\right) a_{1 i 1}\right)^{2}+\left(\sum_{i} \phi_{1 i}\left(L_{1}\right) b_{1 i 1}\right)^{2}} \\
& =\sqrt{\left[\phi_{11}\left(L_{1}\right) a_{11}+\phi_{12}\left(L_{1}\right) a_{12}+L\right]^{2}+\left[\phi_{11}\left(L_{1}\right) b_{11}+\phi_{12}\left(L_{1}\right) b_{12}+L\right]^{2}}, \\
\widehat{w}_{2}\left(L_{2}, t\right) & =\sqrt{\left(\sum_{j} \phi_{2 j}\left(L_{2}\right) a_{2 j 1}\right)^{2}+\left(\sum_{j} \phi_{2 j}\left(L_{2}\right) a_{2 j 1}\right)^{2}} \\
& =\sqrt{\left[\phi_{21}\left(L_{2}\right) a_{21}+\phi_{22}\left(L_{2}\right) a_{22}+L\right]^{2}+\left[\phi_{21}\left(L_{2}\right) b_{21}+\phi_{22}\left(L_{2}\right) b_{22}+L\right]^{2}}, \\
w_{2}\left(L_{2}, t\right) & =\sqrt{\left(\sum_{i}\left[\phi_{1 i}\left(L_{1}\right)+\phi_{1 i}^{\prime}\left(L_{1}\right)\right] a_{1 i 1}+\sum_{j} \phi_{2 j}\left(L_{2}\right) a_{2 j 1}\right)^{2}+\left(\sum_{i}\left[\phi_{1 i}\left(L_{1}\right)+\phi_{1 i}^{\prime}\left(L_{1}\right)\right] b_{1 i 1}+\sum_{j} \phi_{2 j}\left(L_{2}\right) b_{2 j 1}\right)^{2}} .
\end{aligned}
$$

The vibration of the free end of the primary system is the point with largest amplitude, which is the response point to describe the vibration suppression effectiveness. Figure 6 shows the comparison of amplitudes of deflections of the primary beam and absorber beam on frequency band for different structural parameters of magnetic interaction.

From Figures 6(a)-6(e), it can be seen that not only are the resonance bands and nonresonance changed, but also the amplitudes dependent on the magnetic field are changed. For the increase of distance $d$ (reduction of magnetic field), the first nonresonance frequency band increases and then reduces, which conforms to the results for the variations of natural frequencies in Figure 5. From the amplitude-frequency curves of the primary beam shown in Figure 6, the response at the first resonance frequency displays linearity, while nonlinear phenomenon occurs at higher-order resonance frequency, which is induced by the magnetic interaction. Compared to the case without magnetic interaction in Figure 6(f), the resonance peaks for the cases with magnetic interaction are much lower. In addition, for different values of $d$, the system displays hard-spring or soft- spring properties at the high-order resonance frequencies. This phenomenon reveals that the vibration of the primary beam is effectively suppressed because of the nonlinearity coupling of magnetic interaction, especially at resonance frequency band.

\subsection{Width of Frequency Band-Based Design and Evaluation} Criteria. To design the structure and magnetic field, the evaluation criteria are proposed according to the effect and mechanism of vibration suppression of the primary system. Here, we define two criteria to evaluate the vibration suppression effect based on the width of frequency band for vibration suppression.

Firstly, the absorber beam is considered effective when the response amplitude of the primary beam is lower than the case for nonabsorber. Similarly, the magnetic interaction is considered useful when the response amplitude of the primary beam is lower than the case for nonmagnetic connection. Then, the width of frequency bands where the vibration amplitude of primary beam is smaller than the case 

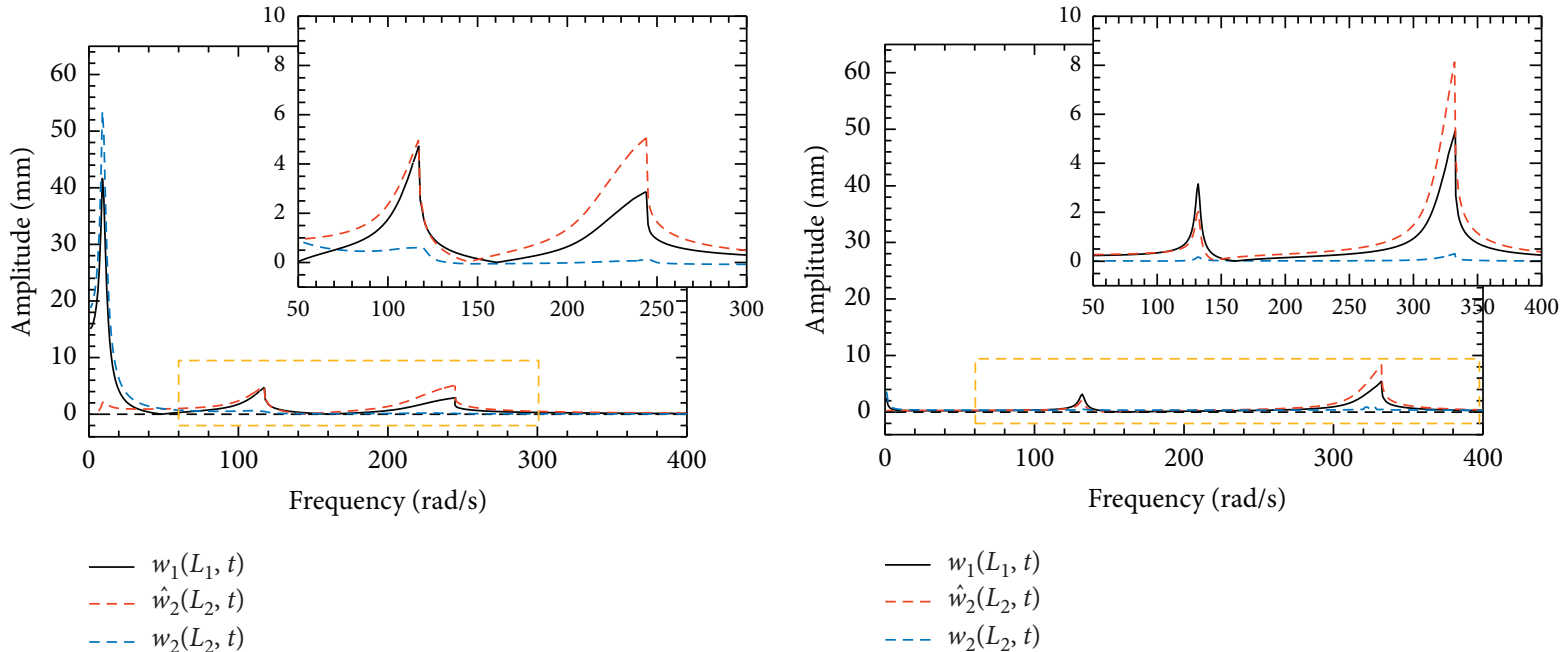

(a)

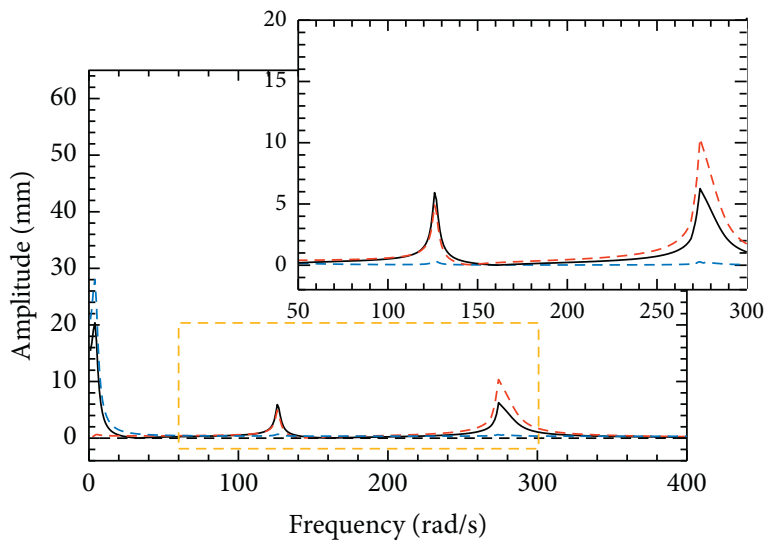

(b)

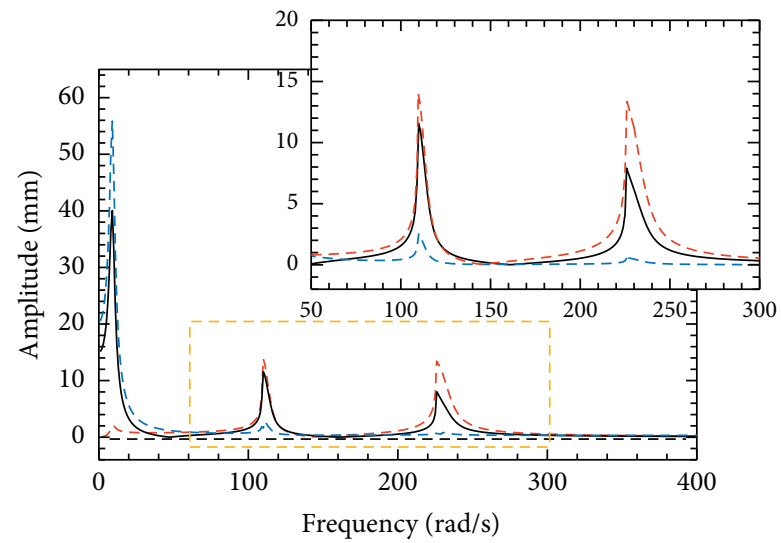

$$
\begin{aligned}
& -w_{1}\left(L_{1}, t\right) \\
& ---\hat{w}_{2}\left(L_{2}, t\right) \\
& ---w_{2}\left(L_{2}, t\right)
\end{aligned}
$$

$$
\begin{aligned}
& -w_{1}\left(L_{1}, t\right) \\
& ---\hat{w}_{2}\left(L_{2}, t\right)
\end{aligned}
$$$$
\text { - - } w_{2}\left(L_{2}, t\right)
$$

(c)

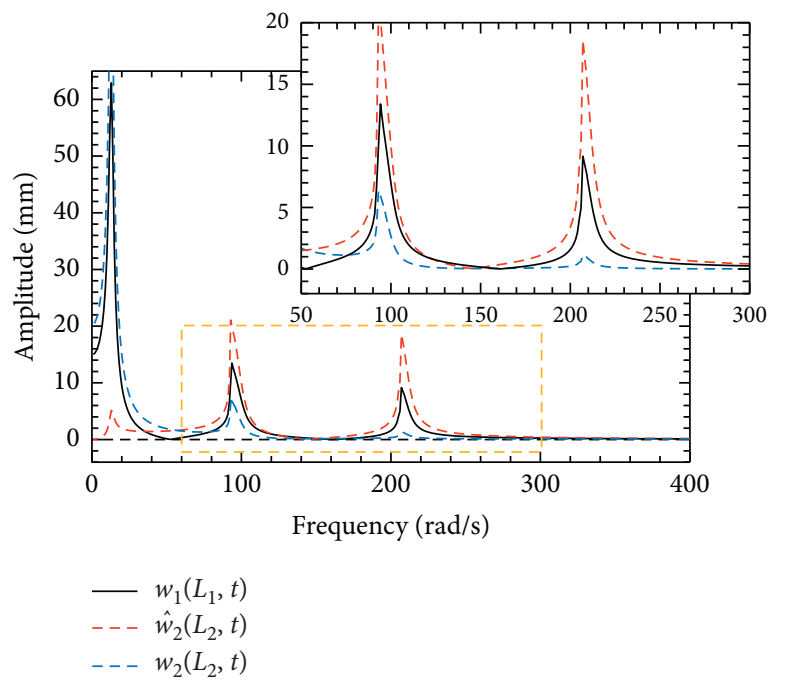

(e)

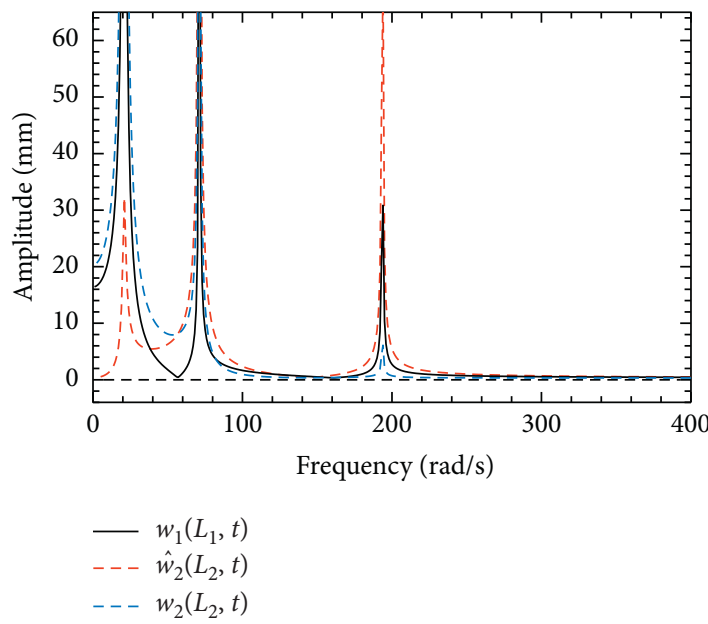

(f)

Figure 6: The amplitude-frequency curves when fixing the force amplitude $f_{0}=0.1 \mathrm{~N}$ for different structural parameters of magnets as (a) $d=5 \mathrm{~mm}$, (b) $d=10 \mathrm{~mm}$, (c) $d=20 \mathrm{~mm}$, (d) $d=30 \mathrm{~mm}$, (e) $d=40 \mathrm{~mm}$, and (f) the case without magnets. 


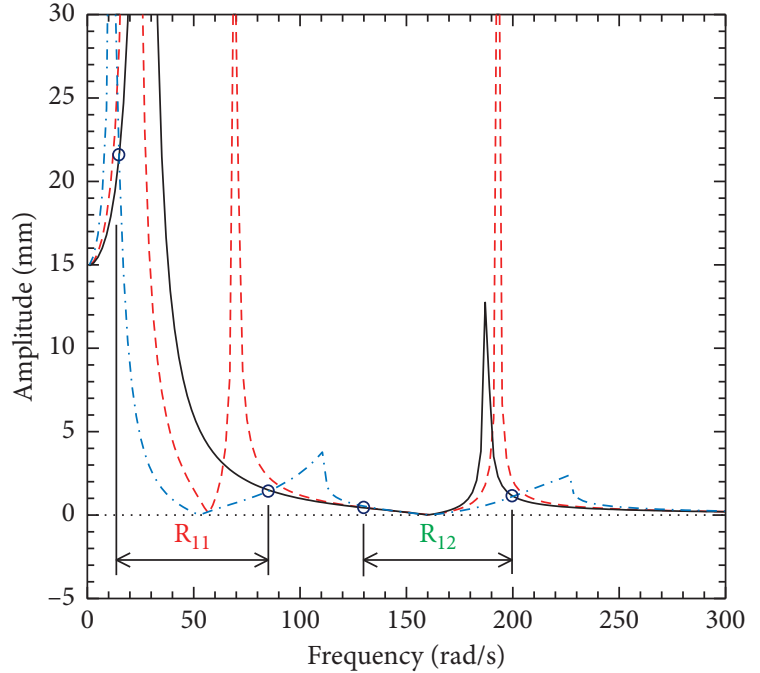

_ Without absorber
_-- Without mag
-.- With mag

(a)

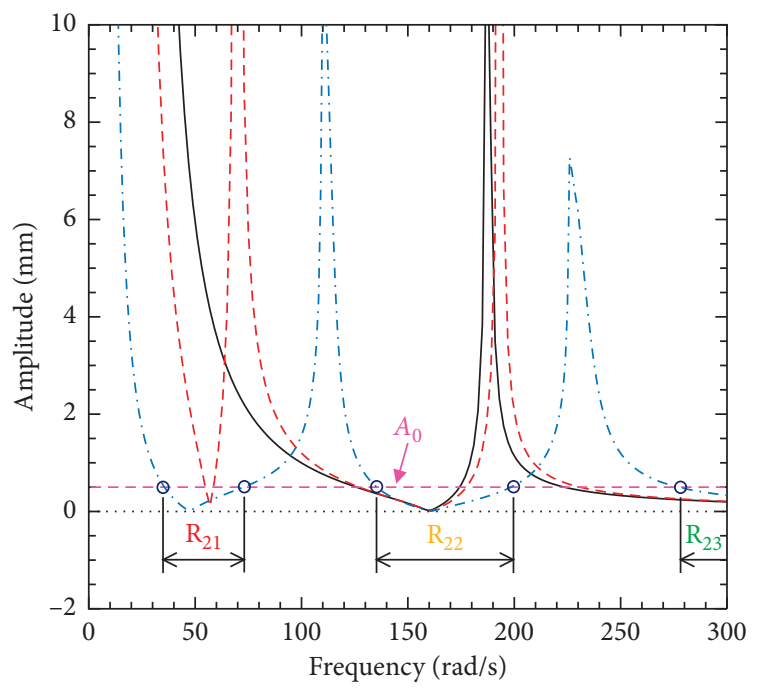

_- Without absorber
- - Without mag
- - With mag

(c)

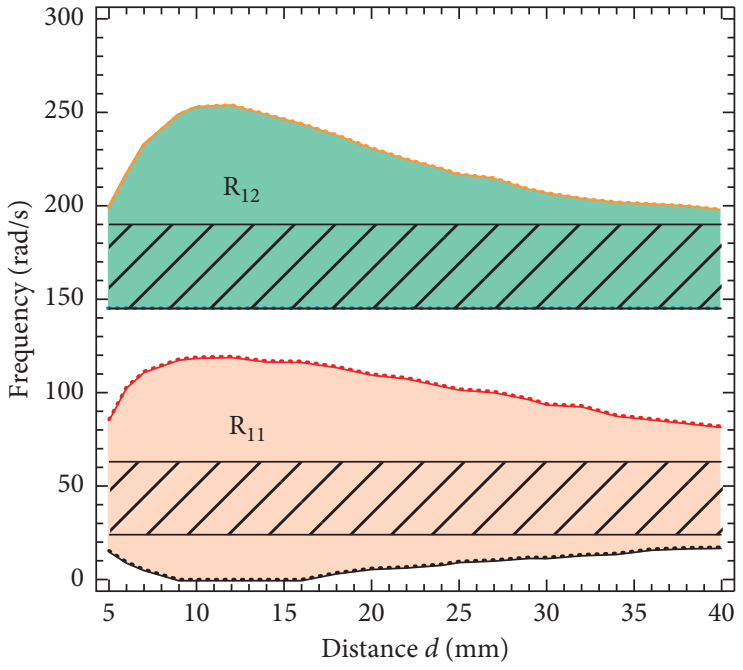

マ W.... With mag

(b)

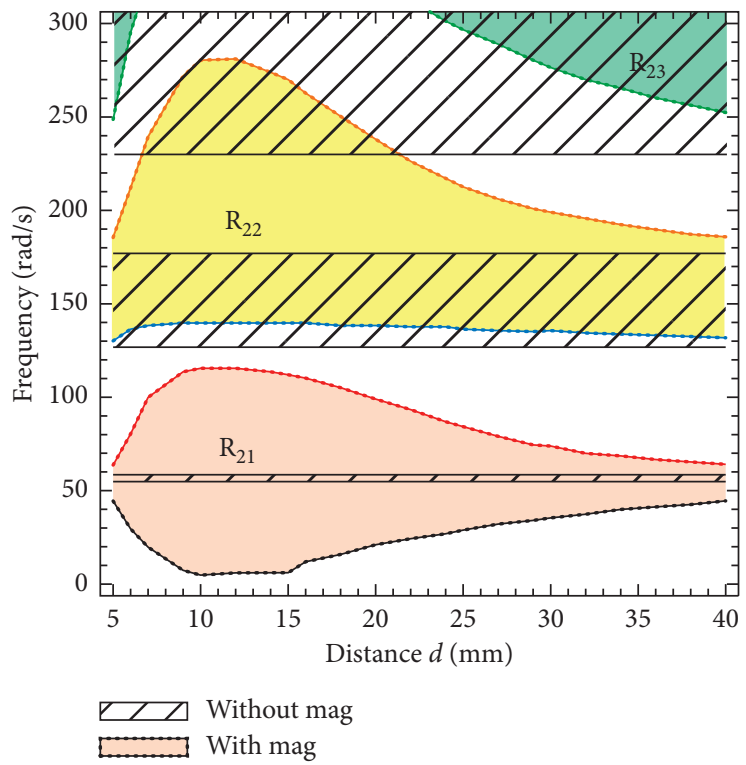

(d)

Figure 7: (a, c) The amplitude-frequency curves for different distances $d$ when fixing the force amplitude $f_{0}=0.1 \mathrm{~N}$; (b, d) frequency bands for vibration absorption and vibration suppression.

without absorber beam or magnetic interaction is expressed as

$$
\mathbf{I}_{1}=\left\{\mathbf{R}_{1} \mid\left\|w_{1}\right\| \leq\left\|w_{0}\right\|\right\}
$$

where $w_{0}$ represents the vibration response of the primary beam for the case without absorber beam or magnetic interaction. The frequency bands obtained by the above criterion (25) show the band for effective vibration absorption.

Secondly, we only consider the requirement for the vibration amplitude of the primary beam rather than the comparison of the primary beam and absorber beam. Thus, we define the effective vibration suppression band when the vibration amplitude of the primary system is less than an acceptable value $A_{0}$ which is required in engineering practices. The width of frequency band for response with amplitude in the predefined value gives the guidance for the design of magnetic interaction. The width of frequency band is expressed as

$$
\mathbf{I}_{2}=\left\{\mathbf{R}_{2} \mid\left\|w_{1}\right\| \leq A_{0}\right\},
$$

where $A_{0}$ is fixed as $A_{0}=0.5 \mathrm{~mm}$ in the following case study. 


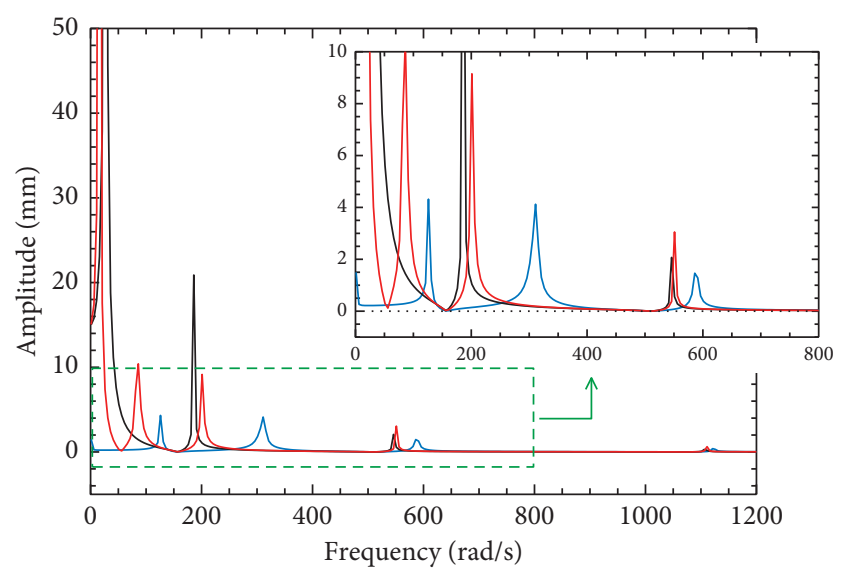

Without absorber
With mag $d=50 \mathrm{~mm}$
With mag $d=12 \mathrm{~mm}$

Figure 8: The comparison of amplitude curves of primary system among cases without absorber and with absorber beam and magnetic interaction for $d=50 \mathrm{~mm}$ and for $d=12 \mathrm{~mm}$.

Figure 7 shows the comparison of amplitude-frequency curves of the primary beam with and without magnetic interaction. The variations of the frequency bands width defined by the vibration absorption criterion (25) and vibration suppression criterion (26) for different distances $d$ are also shown in Figure 7.

From the amplitude-frequency curves shown in Figures 7(a) and 7(c), the comparison between the cases with and without magnets shows the effect of magnetic interaction on the resonance frequencies, resonance peaks, and amplitudes of the primary system. The assembly of absorber beam with magnetic interaction induces an antiresonance frequency band at the original first resonance frequency of the primary beam. At the fundamental frequency, since the response displays linear property, it is difficult to reduce the resonance peak but the antiresonance frequency point can be easily adjusted. In contrast, at the high-order resonance frequency, the antiresonance frequency point is fixed, while the resonance peaks can be significantly suppressed, which is the effect of nonlinearity coupling. From the defined frequency bands by the two criteria (25) and (26) as shown in Figures $7(\mathrm{~b})$ and $7(\mathrm{~d})$, the first vibration suppression/absorption frequency bands are widest for $d \approx 12 \mathrm{~mm}$. The comparison of amplitude-frequency curves among the cases without absorber and with absorber for $d=50 \mathrm{~mm}$ and the optimal value $d=12 \mathrm{~mm}$ is shown in Figure 8 .

From the amplitude-frequency curves in Figure 8, for $d=12 \mathrm{~mm}$, the beginning frequency of effective vibration suppression band is close to zero and the resonance peaks due to the negative stiffness property by the magnetic interaction. In addition, the values of second and third resonance peaks for $d=12 \mathrm{~mm}$ are nearly equal and are much lower than the other two cases, which is the benefit brought by the nonlinearity coupling between the primary and absorber beams. Thus, the magnetic coupling absorber beam has adjustable property for nonresonance frequency band and also has significant effect on the vibration suppression, especially for low-frequency resonance.

4.4. Discussions on the Superharmonic Resonance Induced by Nonlinearity. Since the nonlinearity induced by the magnetic interaction is obvious at the equilibrium, it would bring high-order harmonic components, which would not occur in linear system. Therefore, we consider the high-order harmonic components of responses as

$$
\begin{aligned}
q_{1 i}(t)= & a_{1 i_{1}} \cos (\Omega t)+b_{1 i_{1}} \sin (\Omega t)+a_{1 i_{2}} \cos (3 \Omega t) \\
& +b_{1 i_{2}} \sin (3 \Omega t) \\
q_{2 j}(t)= & a_{2 j_{1}} \cos (\Omega t)+b_{2 j_{1}} \sin (\Omega t)+a_{1 i_{2}} \cos (3 \Omega t) \\
& +b_{1 i_{2}} \sin (3 \Omega t) .
\end{aligned}
$$

By substituting the solution into dynamical equation and separating harmonic components for $\cos (\Omega t), \sin (\Omega t)$, $\cos (3 \Omega t)$, and $\sin (3 \Omega t)$, there are $2 \times 2 \times(K+N)$ algebraic equations. The amplitudes of superharmonic components for different structural parameters and the comparison of amplitude-frequency curves between the cases considering superharmonic components are shown in Figure 9.

From Figure 9, the superharmonic components would occur due to the nonlinearity from magnetic interaction. Also, with the increase of excitation amplitude, the superharmonic components are growing up. But, with the comparison of the amplitudes between the primary- and superharmonic components, the values of superharmonic amplitudes are much smaller than the primary-harmonic ones. The primary-harmonic amplitudes for the case considering the superharmonic components have little error compared to the case without considering superharmonic components. Thus, the structural parameters of absorber beam and magnetic field can be designed according to the two proposed criteria (25) and (26) for vibration suppression requirements and the superharmonic vibrations can be ignored in practices.

4.5. Experimental Setup and Results. The corresponding experimental prototype of the magnetic interaction based vibration absorber for continuous beam is shown in Figure 10. The experimental setup is shown in Figure 10(a). The magnetic field is shown in Figure 10(b). The absorber beam with different strengths of magnetic coupling is shown in Figure 10(c). The structures are mounted on a vibration table (APS 400). A signal generator (RIGOL DG1022U) controls the structures. An oscilloscope (Tektronix TDS 2002C) measures and stores the output voltage signals. An absorber beam, which has much smaller mass than the primary beam, is introduced and coupled on the primary beam by constraint and magnetic interaction. Two pairs of magnets are assembled on the primary and absorber beams.

The experiment's results are shown in Figure 11. The left and right parts of the figure show the vibration responses and output voltage for the main structure, respectively. 


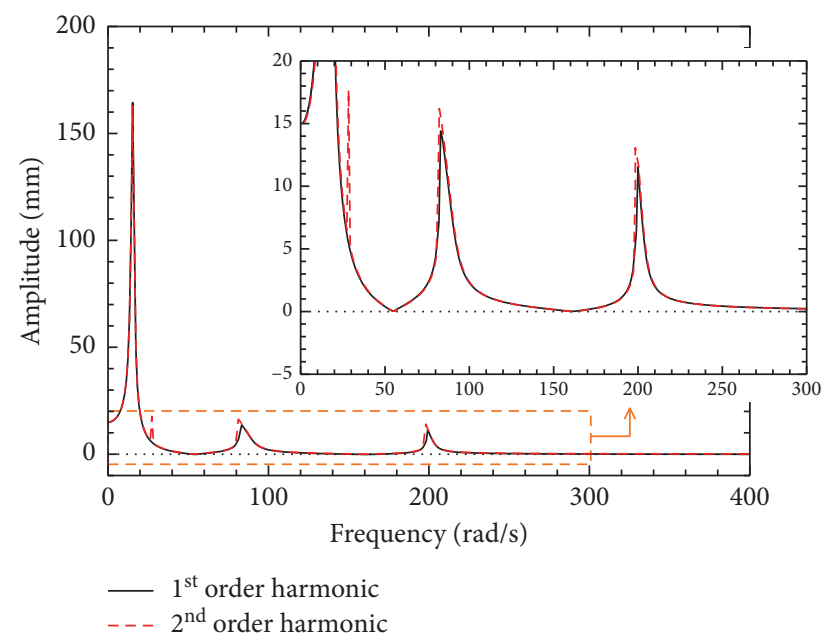

Figure 9: The amplitude curves of primary system considering the first-harmonic and second-harmonic components.

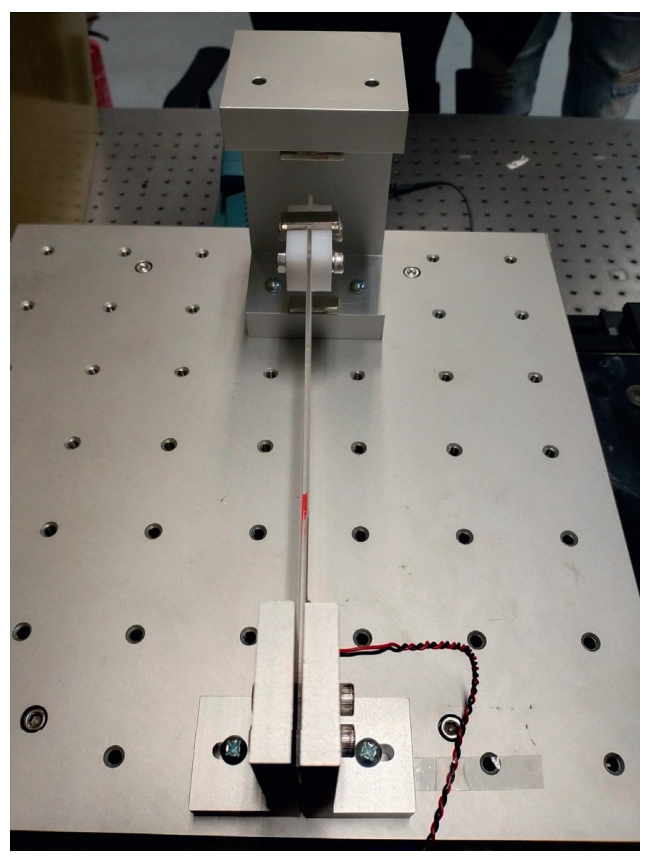

(a)
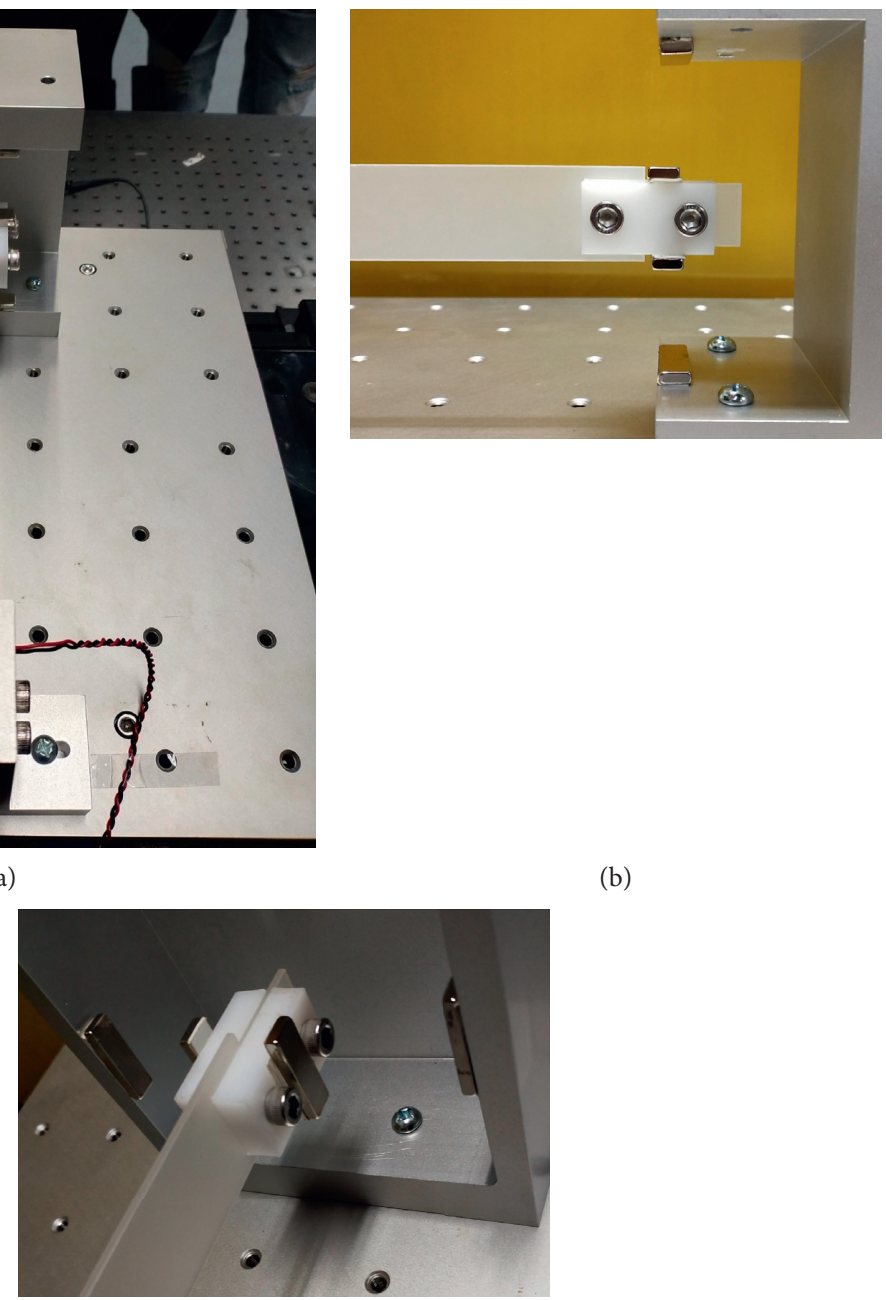

(b)

(c)

Figure 10: (a) The experimental setup; (b) the magnetic field; (c) the absorber beam with different strengths of magnetic coupling. 


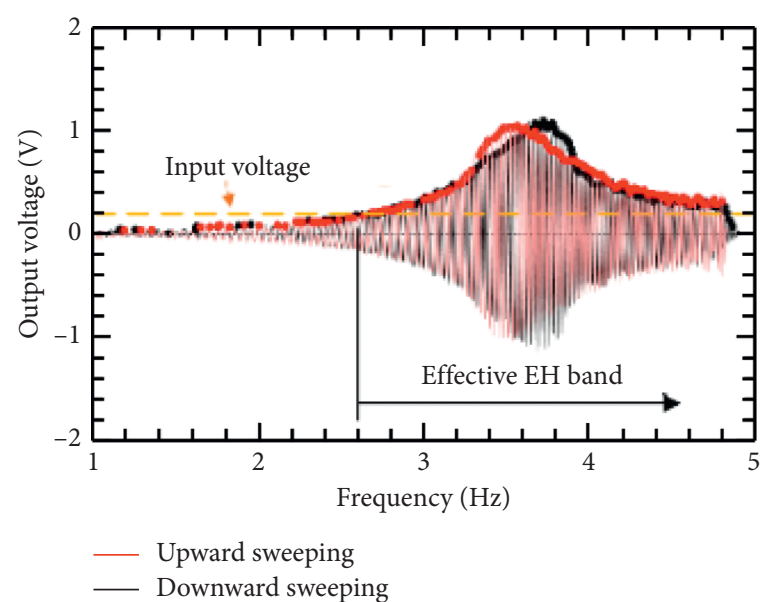

(a)

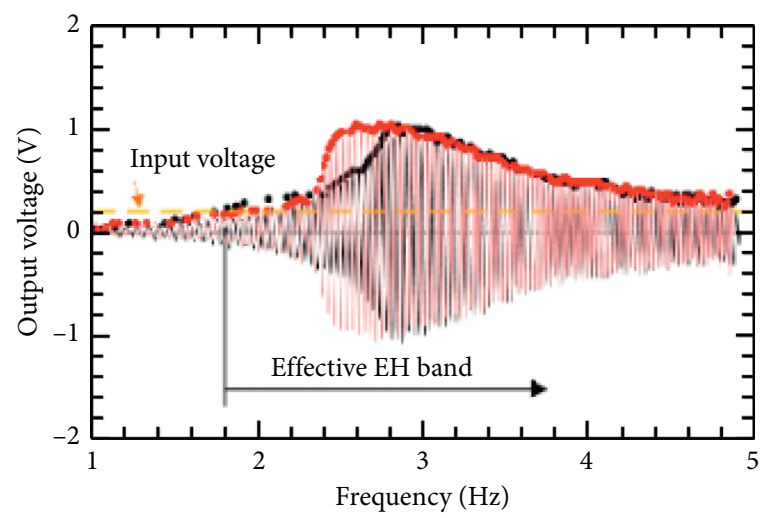

— Upward sweeping

— Downward sweeping

(c)

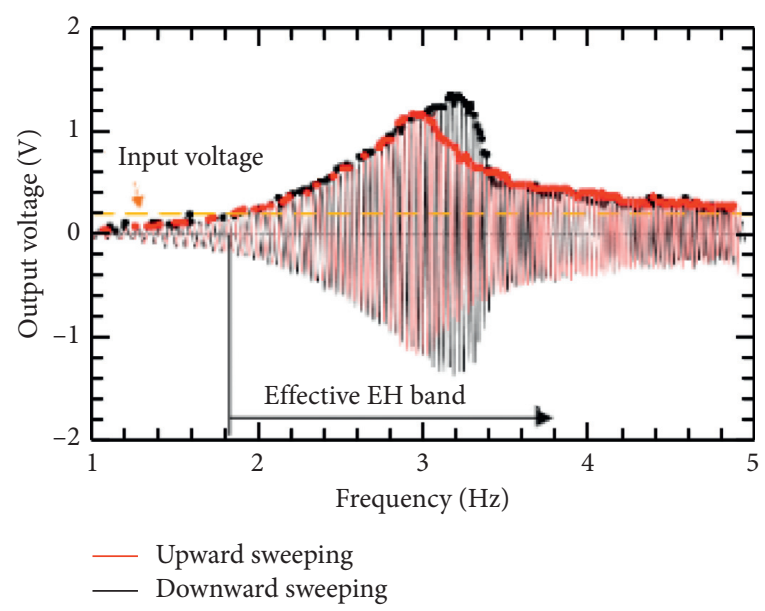

(e)

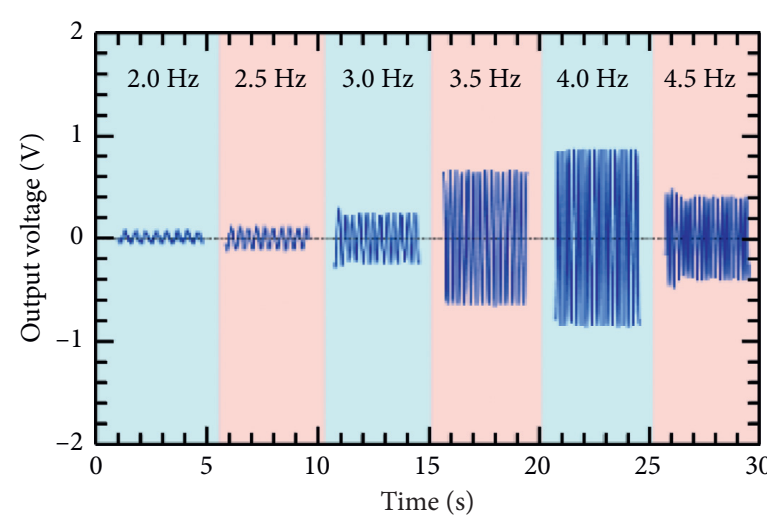

(b)

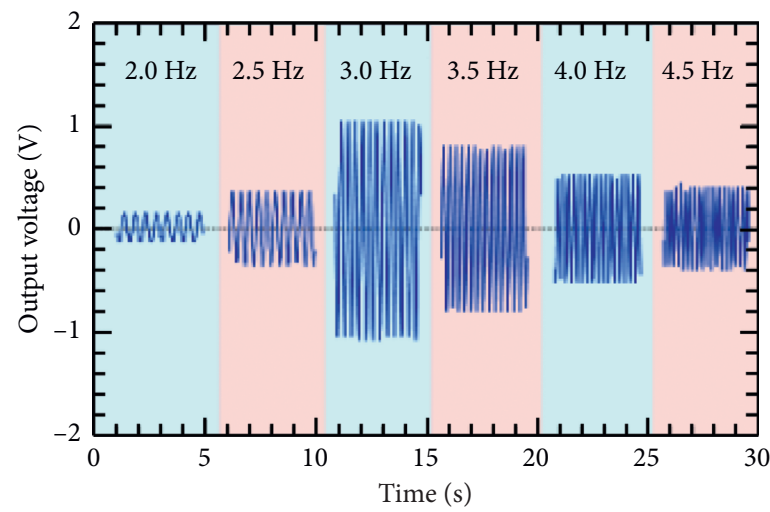

(d)

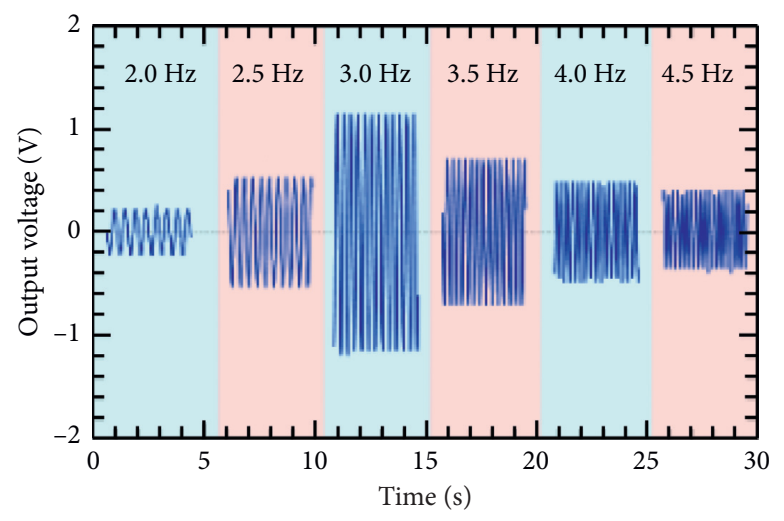

(f)

FIgURE 11: For time-lasting external excitation, (a) vibration responses and (b) output voltage for the main structure for weak magnetic interactions; (c) vibration responses and (d) output voltage for the main structure for middle strength magnetic interactions; (e) vibration responses and (f) output voltage for the main structure for strong magnetic interactions.

Figures 11(a) and 11(b) correspond to weak magnetic interactions, $11(\mathrm{c})$ and $11(\mathrm{~d})$ correspond to middle magnetic interactions, and 11(e) and 11(f) correspond to strong magnetic interactions. The strength of magnetic interaction can be adjusted by changing the magnetic parameters in the connection region. The frequency-sweep tests with a linear sweep rate of $0.1 \mathrm{~Hz} \cdot \mathrm{s}^{-1}$ in the frequency range between $1 \mathrm{~Hz}$ and $5 \mathrm{~Hz}$ are carried out with upward and downward 
sweeping. In Figure 11(a), the resonant frequency of the main structure for weak magnetic interactions is about $3.6 \mathrm{~Hz}$ and the largest output voltage reaches $1.1 \mathrm{~V}$. In Figure 11(c) corresponding to middle strength magnetic interactions, the peak voltage lies between 2.4 and $2.8 \mathrm{~Hz}$ and reaches $1.2 \mathrm{~V}$ for the two times of sweeping, performing softspring property. The output signal shown in Figure 11(e), corresponding to strong magnetic interactions, is beyond $1.4 \mathrm{~V}$, which is, respectively, $27 \%$ and $17 \%$ larger than the voltages generated by lower-strength magnetic interactions. Although the frequency band in Figure 11(e) is almost the same as the one in Figure 11(c), the peak frequency is between 3 and $3.4 \mathrm{~Hz}$, wider than the second case due to its hard-spring characteristic. Figures 11(b), 11(d), and 11(f) show the voltages generated by the magnetic coupled absorber structures under fixed-frequency excitation, which agrees with the results in frequency-sweeping results. This experiment proves that adjusting the magnetic parameters in the connection region can change the absorber's resonance frequency to match the frequency and damping of main structure effectively.

\section{Conclusions}

In this paper, a continuous beam as absorber is assembled on a continuous primary structure to suppress the vibration. The absorber beam is coupled on continuous beam by crosssection-fixed-connection and magnetic interaction; thus the mutual effect of the primary beam and absorber beam contains both shear force and bending moment. The following conclusions can be drawn based on our results and discussions:

(a) Based on the interaction force model of magnets and the dynamical model of the system, discrete dynamic equation for each order of the system is obtained. For appropriate magnetic interaction field, the fundamental frequency of the system can be reduced closely to zero, and the second and third natural frequencies increase initially and then reduce. Thus, a wide frequency band for nonresonance exists from zero.

(b) With the response solutions solved, since the magnetic interaction is local and nonlinear, the values of the resonance peaks can be suppressed much lower than the case without magnetic interaction for appropriate magnetic structural parameters. The amplitude-frequency curves for different structural parameters reveal that the coupling interaction, especially the bending moment, applied on the primary beam by the absorber beam remarkably suppresses the vibration responses of the primary beam. Since the effect of the absorber beam is amplified by the motion of the primary beam in each mode, the mass of absorber beam is much tinier than the primary beam.

(c) According to the comparison of amplitude-frequency curves for the cases with/without magnetic interaction, two criteria are defined, which describe the improvement effect of the nonlinearity coupling induced by magnetic interaction. The effective vibration frequency bands are extended without increasing the absorber mass.

In conclusion, for the proposed absorber beam with the magnetic coupling and assembly, remarkable vibration suppression can be obtained. The responses and design principle of the absorber beam with magnetic interaction are obtained based on the modelling of system and magnetic interaction. The proposed vibration suppression method for resonance vibration in wide frequency band has significant applications as flexible aim for continuous structure in the fields of manufacturing and aerospace.

\section{Appendix}

This appendix presents the structural parameters and coefficients in the theoretical analysis and simulation and natural frequencies for different truncation orders.

Assuming the primary beam and absorber beam are both acrylic materials, the structural parameters are fixed and shown in Table 1.

\section{Nomenclature}

$w_{1}$ : Vibration of the primary system

$\widehat{w}_{2}$ : Deflection vibration of the absorber beam

$f(t)$ : Force excitation

$\xi_{1}$ : Coordinate for the primary beam

$\widetilde{\Omega}_{h}:$ Natural frequencies of the system

$\omega_{2 n}$ : Natural frequencies of absorber beam

$w_{2}$ : Displacement of the absorber beam

$\theta_{1}$ : Angle of rotation of primary beam

$f: \quad$ Force excitation amplitude

$\xi_{2}$ : Coordinate for absorber beam

$\Omega_{1 k}$ : Natural frequencies of primary beam

$\Omega$ : Excitation frequency.

\section{Data Availability}

The data used to support the findings of this study are available from the corresponding author upon request.

\section{Conflicts of Interest}

The authors declare that there are no conflicts of interest regarding the publication of this paper.

\section{References}

[1] S. Keye, R. Keimer, and S. Homann, "A vibration absorber with variable eigenfrequency for turboprop aircraft," Aerospace Science and Technology, vol. 13, no. 4-5, pp. 165-171, 2009.

[2] X. Huang, Z. Su, and H. Hua, "Application of a dynamic vibration absorber with negative stiffness for control of a marine shafting system," Ocean Engineering, vol. 155, pp. 131-143, 2018.

[3] Z. Q. Zheng, P. Huang, T. Yao, and Z. Y. Chang, "Dynamics analysis of energy absorbing device based on damped 
dynamics vibration absorber," Applied Mechanics and Materials, vol. 764-765, pp. 254-258, 2015.

[4] N. D. Anh, H. Matsuhisa, L. D. Viet, and M. Yasuda, "Vibration control of an inverted pendulum type structure by passive mass-spring-pendulum dynamic vibration absorber," Journal of Sound and Vibration, vol. 307, no. 1-2, pp. 187-201, 2007.

[5] S. S. Sun, H. X. Deng, H. P. Du et al., "A compact variable stiffness and damping shock absorber for vehicle suspension," IEEE/ASME Transactions on Mechatronics, vol. 20, no. 5, pp. 1-9, 2015.

[6] Y. Starosvetsky and O. V. Gendelman, "Attractors of harmonically forced linear oscillator with attached nonlinear energy sink. II: optimization of a nonlinear vibration absorber," Nonlinear Dynamics, vol. 51, no. 1-2, pp. 47-57, 2008.

[7] J. Zhou, X. Wang, D. Xu, and S. Bishop, "Nonlinear dynamic characteristics of a quasi-zero stiffness vibration isolator with cam-roller-spring mechanisms," Journal of Sound and Vibration, vol. 346, pp. 53-69, 2015.

[8] X. Wang, H. Liu, Y. Chen, and P. Gao, "Beneficial stiffness design of a high-static-low-dynamic-stiffness vibration isolator based on static and dynamic analysis," International Journal of Mechanical Sciences, vol. 142-143, pp. 235-244, 2018.

[9] R. A. Ibrahim, "Recent advances in nonlinear passive vibration isolators," Journal of Sound and Vibration, vol. 314, no. 3-5, pp. 371-452, 2008.

[10] X. Sun, J. Xu, F. Wang, and L. Cheng, "Design and experiment of nonlinear absorber for equal-peak and de-nonlinearity," Journal of Sound and Vibration, vol. 449, pp. 274-299, 2019.

[11] R. Viguié and G. Kerschen, "Nonlinear vibration absorber coupled to a nonlinear primary system: a tuning methodology," Journal of Sound and Vibration, vol. 326, no. 3-5, pp. 780-793, 2009.

[12] M. Sayed and M. Kamel, "1:2 and 1:3 internal resonance active absorber for non-linear vibrating system," Applied Mathematical Modelling, vol. 36, no. 1, pp. 310-332, 2012.

[13] D. Zulli and A. Luongo, "Nonlinear energy sink to control vibrations of an internally nonresonant elastic string," Meccanica, vol. 50, no. 3, pp. 781-794, 2015.

[14] D. Yu, Y. Liu, H. Zhao, G. Wang, and J. Qiu, "Flexural vibration band gaps in Euler-Bernoulli beams with locally resonant structures with two degrees of freedom," Physical Review B, vol. 73, Article ID 064301, 2006.

[15] G. Wang, J. Wen, and X. Wen, "Quasi-one-dimensional phononic crystals studied using the improved lumped-mass method: application to locally resonant beams with flexural wave band gap," Physical Review B, vol. 71, no. 10, Article ID 104302, 2005.

[16] J. Zhou, K. Wang, D. Xu, and H. Ouyang, "Multi-low-frequency flexural wave attenuation in Euler-Bernoulli beams using local resonators containing negative-stiffness mechanisms," Physics Letters A, vol. 381, no. 37, pp. 3141-3148, 2017.

[17] J. Zhou, L. Dou, K. Wang, D. Xu, and H. Ouyang, "A nonlinear resonator with inertial amplification for very low-frequency flexural wave attenuations in beams," Nonlinear Dynamics, vol. 96, no. 1, pp. 647-665, 2019.

[18] T. Asami and O. Nishihara, "Analytical solutions to $H_{\infty}$ and $\mathrm{H}_{2}$ optimization of dynamic vibration absorbers attached to damped linear systems," Transactions of the Japan Society of Mechanical Engineers, vol. 67, pp. 597-603, 2002.

[19] T. Detroux, G. Habib, L. Masset, and G. Kerschen, "Performance, robustness and sensitivity analysis of the nonlinear tuned vibration absorber," Mechanical Systems and Signal Processing, vol. 60-61, pp. 799-809, 2015.

[20] L. Dell'Elce, E. Gourc, and G. Kerschen, "A robust equal-peak method for uncertain mechanical systems," Journal of Sound and Vibration, vol. 414, pp. 97-109, 2018.

[21] P. Soltani and G. Kerschen, "The nonlinear piezoelectric tuned vibration absorber," Smart Materials and Structures, vol. 24, Article ID 075015, 2015.

[22] Y. L. Cheung, W. O. Wong, and L. Cheng, "Design optimization of a damped hybrid vibration absorber," Journal of Sound and Vibration, vol. 331, no. 4, pp. 750-766, 2012.

[23] T. Aida, S. Toda, N. Ogawa, and Y. Imada, "Vibration control of beams by beam-type dynamic vibration absorbers," Journal of Engineering Mechanics, vol. 118, no. 2, pp. 248-258, 1992.

[24] M. H. Tso, J. Yuan, and W. O. Wong, "Suppression of random vibration in flexible structures using a hybrid vibration absorber," Journal of Sound and Vibration, vol. 331, no. 5, pp. 974-986, 2012.

[25] B. Noori and A. Farshidianfar, "Optimum design of dynamic vibration absorbers for a beam, based on $\mathrm{H}_{\infty}$ and $\mathrm{H}_{2}$ Optimization," Archive of Applied Mechanics, vol. 83, no. 12, pp. 1773-1787, 2013.

[26] Y. Hua, W. Wong, and L. Cheng, "Optimal design of a beambased dynamic vibration absorber using fixed-points theory," Journal of Sound and Vibration, vol. 421, pp. 111-131, 2018.

[27] A. O. Oyelade, Z. Wang, and G. Hu, "Dynamics of 1D massspring system with a negative stiffness spring realized by magnets: theoretical and experimental study," Theoretical and Applied Mechanics Letters, vol. 7, no. 1, pp. 17-21, 2017.

[28] X. Sun, H. Zhang, W. Meng, R. Zhang, K. Li, and T. Peng, "Primary resonance analysis and vibration suppression for the harmonically excited nonlinear suspension system using a pair of symmetric viscoelastic buffers," Nonlinear Dynamics, vol. 94, no. 2, pp. 1243-1265, 2018.

[29] C. Liu, Q. Ding, Q. Gong, C. Ma, and S. Yue, "Axial control for nonlinear resonances of electrostatically actuated nanobeam with graphene sensor," Applied Mathematics and Mechanics, vol. 38, no. 4, pp. 527-542, 2017.

[30] X. Sun, F. Wang, and J. Xu, "Nonlinear piezoelectric structure for ultralow-frequency band vibration energy harvesting with magnetic interaction," International Journal of Precision Engineering and Manufacturing-Green Technology, vol. 6, no. 4, pp. 671-679, 2019.

[31] G. Akoun and J. P. Yonnet, "3D analytical calculation of the forces exerted between two cuboidal magnets," IEEE Transactions on Magnetics, vol. 45, no. 10, pp. 3969-3972, 2009.

[32] H. Allag, J. Yonnet, and M. E. H. Latreche, "Analytical calculation of the torque exerted between two perpendicularly magnetized magnets," IEEE Transactions on Magnetics, vol. 57, pp. 828-834, 2011. 